\title{
Stochastic gravitational waves from cosmic string loops in scaling
}

\section{Christophe Ringeval $^{a}$ and Teruaki Suyama ${ }^{b}$}

\author{
${ }^{a}$ Centre for Cosmology, Particle Physics and Phenomenology, Institute of Mathematics and \\ Physics, Louvain University, 2 Chemin du Cyclotron, 1348 Louvain-la-Neuve, Belgium \\ ${ }^{b}$ Research Center for the Early Universe (RESCEU), University of Tokyo, Hongo, 7-3-1 \\ Bunkyo-ku, Tokyo 113-0033, Japan \\ E-mail: christophe.ringeval@uclouvain.be, suyama@resceu.s.u-tokyo.ac.jp
}

\begin{abstract}
If cosmic strings are formed in the early universe, their associated loops emit gravitational waves during the whole cosmic history and contribute to the stochastic gravitational wave background at all frequencies. We provide a new estimate of the stochastic gravitational wave spectrum by considering a realistic cosmological loop distribution, in scaling, as it can be inferred from Nambu-Goto numerical simulations. Our result takes into account various effects neglected so far. We include both gravitational wave emission and backreaction effects on the loop distribution and show that they produce two distinct features in the spectrum. Concerning the string microstructure, in addition to the presence of cusps and kinks, we show that gravitational wave bursts created by the collision of kinks could dominate the signal for wiggly strings, a situation which may be favoured in the light of recent numerical simulations. In view of these new results, we propose four prototypical scenarios, within the margin of the remaining theoretical uncertainties, for which we derive the corresponding signal and estimate the constraints on the string tension put by both the LIGO and European Pulsar Timing Array (EPTA) observations. The less constrained of these scenarios is shown to have a string tension $G U \leq 7.2 \times 10^{-11}$, at $95 \%$ of confidence. Smooth loops carrying two cusps per oscillation verify the two-sigma bound $G U \leq 1.0 \times 10^{-11}$ while the most constrained of all scenarios describes very kinky loops and satisfies $G U \leq 6.7 \times 10^{-14}$ at $95 \%$ of confidence.
\end{abstract}

Keywords: Cosmic Strings, Gravitational Waves, Loops 


\section{Contents}

1 Introduction $\quad 1$

2 Gravitational wave sources $\quad 5$

2.1 Basic assumptions 5

$\begin{array}{lll}2.2 & \text { Cusps, kinks and collisions } & 7\end{array}$

2.3 Single loop energy and spectral density 8

3 Stochastic gravitational wave spectrum $\quad 9$

3.1 Unified source functions and scaling variables 11

$\begin{array}{lll}3.2 & \text { Loop visibility domains } & 12\end{array}$

$\begin{array}{lll}3.3 & \text { Results } & 15\end{array}$

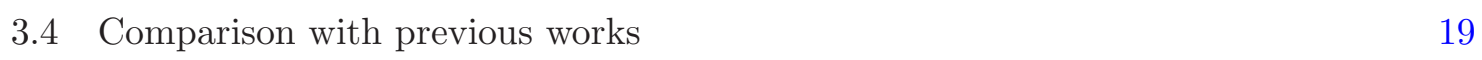

4 Loop microstructure and constraints $\quad 21$

4.1 Gravitational wave emission bound 21

4.2 Prototypical models of string microstructure 22

$\begin{array}{lll}4.3 & \text { Observational constraints } & 23\end{array}$

4.3.1 LIGO and EPTA data 23

4.3.2 Constraints on the string tension 24

5 Conclusion

\section{Introduction}

Cosmic strings are topological line defects expected to be formed during phase transitions in the early Universe $[1,2]$. They could also be mimicked by more fundamental objects, such as D1-brane of F-strings, directly stemming from String Theory and referred to as cosmic superstrings $[3,4]$. In the cosmological context, once formed, a string network relaxes towards a cosmological attractor that exhibits universal statistical properties [5-11]. For instance, the number of strings crossing a Hubble radius remains stationary for the rest of the cosmic history, this is the so-called "scaling" regime. For Nambu-Goto strings, this implies that all observable predictions depend on one parameter only which is the string energy density $U$ (also equals to the string tension), a quantity related to the energy scale at which the strings have been formed. The determination of a Nambu-Goto network's scaling properties in Friedmann-Lemaitre-Robertson-Walker spacetime (FLRW) is a non-trivial issue and has been the subject of numerical investigations in the last thirty years [12-15]. These works have shown that the long strings are indeed able to reach the cosmological attractor by loosing energy under the form of loops, these ones loosing energy in turn under the form of smaller loops and gravitational waves $(\mathrm{GW})$. As a result, it has been known for a long time that cosmic strings could potentially be a dominant source for the Universe stochastic gravitational wave background [16-20]. This picture could however be challenged by the Abelian Higgs strings in which strings can decay by particle emission instead of GW [21-24]. In the following, we will be focused on Nambu-Goto strings only although our modelling could be extended to include decay channels others than GW. 
In an expanding Universe, the distribution of cosmic string loops, i.e. the number density of loops with respect to their size, reaches a scaling regime, a result first shown in Ref. [25] and soon after confirmed in Refs. [26, 27]. However, most of the works having estimated the stochastic gravitational wave spectrum from loops have assumed utterly simplified loop distribution, or loop production function, usually postulated to be a Dirac function peaked at a length equal to some given fraction of the horizon size [28-36]. In the meanwhile, more recent Nambu-Goto simulations presented in Ref. [37] have independently recovered the power-law shape of the loop distribution originally found in Ref. [25] thereby giving a robust picture of the loop scaling regime on the length scales reachable in numerical simulations. The authors of Ref. [37] have also provided a new estimation of the gravitational wave spectrum stemming from their loop distribution. As we discuss in section 3.4, when the additional effects we are considering are switched off, our spectrum is of similar shape and amplitude compared to the one of Ref. [37].

The shape of the loop distribution on the length scales probed by numerical simulations is however not enough to uniquely determine their associated stochastic GW spectrum. Some theoretical uncertainties remain.

Firstly, gravitational wave emission impacts the scaling loop distribution. All loops shrink by loosing energy under the form of GW and the loop distribution ends up being modified when such a process becomes faster than the other mechanisms at work in a string network (production from string self-intersections and loop fragmentation). The emitted $\mathrm{GW}$ power is given by $P_{\mathrm{gw}}=\Gamma G U^{2}$ such that $\mathrm{GW}$ evaporation dominates for loops of size $\ell<\Gamma G U t, G$ being the Newton constant, $t$ the cosmic time and $\Gamma$ a numerical constant estimated to be $\Gamma=\mathcal{O}(50)[38,39]$. For this reason, this length scale is the one under which numerical simulations cannot be trusted for cosmological purposes. For the maximal allowed values of $G U=10^{-7}$, this regime appears for loops smaller than a millionth the size of the horizon [40-42]. In the following, we define

$$
\gamma_{\mathrm{d}} \equiv \Gamma G U
$$

the gravitational wave emission length scale, measured in unit of $t$.

Secondly, the gravitational wave signal emitted by a single loop mostly depends on the string microstructure, and, as shown by Damour and Vilenkin in Ref. [43], the spectrum at high frequency is dominated by the transient appearance of "cusps" in the shape of the string, and more generally on piece of strings approaching the speed of light [44]. Its amplitude scales as $\omega^{-4 / 3}, \omega$ being the GW angular frequency. As a result, the signal amplitude depends on the number of cusps appearing per loop oscillation. GW can also be produced from "kinks" in the shape of the string, with an amplitude varying however as $\omega^{-5 / 3}$ [43]. Therefore, kinks are expected to be a sub-dominant contribution provided their number remains less, or comparable, to the number of cusps. Unfortunately, the number of cusps and kinks per loop oscillation is not yet known and cannot be straightforwardly derived from numerical simulations. These numbers indeed depend on the so-called gravitational wave backreaction, an effect which is not included in the simulations. Kinks appear in pairs from string collisions, then propagate at the speed of light in opposite directions, and have a tendency to accumulate over the cosmological evolution. All Nambu-Goto simulations have shown that the loops are extremely wiggly, i.e. filled with kinks $[13,14]$. However, by emitting gravitational waves, one expects backreaction to wash-out the string microstructure under some given length scale thereby rendering the kinky loops smoother, a mechanism known to favour the appearance of cusps [45]. This smoothing mechanism is the standard lore but has been recently challenged 
in Refs. $[46,47]$ in which the effect of gravitational wave backreaction on the shape of the loops has been explicitly estimated for various loop shapes. Some loops become smoother, as expected, but others can actually remain filled with kinks.

Thirdly, in addition to modify the number of kinks and cusps per loop oscillations, gravitational wave backreaction can also modify the scaling loop distribution. Because very small loops are created from the microstructure of larger strings and larger loops, gravitational wave backreaction is expected to switch off loop production mechanisms in a string network under a given length scale. Another simplifying assumption made in all the works having estimated the stochastic gravitational wave spectrum so far is that the scale of gravitational wave backreaction matches the scale of gravitational wave emission, $\gamma_{\mathrm{d}}$. However, this is usually not the case. As shown in Refs. [48-50], gravitational wave backreaction on kinky strings may appear at a length scale $\ell_{\mathrm{c}}$, which can be much smaller than $\ell_{\mathrm{d}}=\gamma_{\mathrm{d}} t$. In the following, we denote by $\gamma_{\mathrm{c}} \equiv \ell_{\mathrm{c}} / t$ the backreaction length scale, measured in unit of $t$. From Ref. [50], it has been estimated to be

$$
\gamma_{\mathrm{c}} \equiv \Upsilon(G U)^{1+2 \chi}
$$

in which $\Upsilon=\mathcal{O}(20)$. The parameter $\chi$ is related to the tangent vector correlations along the strings and can be read-off from the shape of the loop distribution on large scales, and thus can be numerically determined from Nambu-Goto simulations ${ }^{1}$.

In this work, we tackle various of these remaining theoretical uncertainties and provide a new estimate of the stochastic gravitational wave spectrum generated by cosmic string loops. We include both the effect of gravitational wave emission and gravitational wave backreaction on the scaling loop distribution. In order to do so, we have followed the method of Refs. $[52,53]$ and we solve a two-dimensional Boltzmann equation to determine the loop number density distribution $\mathcal{F}$ at any redshift $z$ :

$$
\mathcal{F}(\gamma, z) \equiv \frac{\mathrm{d} n}{\mathrm{~d} \ell}, \quad \gamma(\ell, z) \equiv \frac{\ell}{t(z)},
$$

$n$ being the number of loops per unit volume. This Boltzmann equation includes gravitational wave emission and a scaling loop production function inferred from the Nambu-Goto simulations of Ref. [25]. As shown in Ref. [54], over the length scales accessible within Nambu-Goto numerical simulations, this loop production function matches the one predicted by Polchinski and Rocha in Refs. [55, 56]. Therefore, we use the Polchinski-Rocha (PR) model to extrapolate the loop production function down to the scales at which gravitational wave backreaction appears. As discussed in Ref. [52], the details on how backreaction smooths the strings is irrelevant for the number of loops, only the scale at which gravitational backreaction shows up, i.e. the value of $\gamma_{c}$, ends up having an observable effect. This matching with the PR model allows us to unambiguously determine the parameter $\chi$ of Eq. (1.2). One gets

$$
\chi_{\mathrm{M}}=0.295_{-0.04}^{+0.03}, \quad \chi_{\mathrm{R}}=0.200_{-0.10}^{+0.07},
$$

for the matter and radiation era, respectively. The central value is the best fit to the PR model while the quoted errors are an overly safe estimate of all the possible numerical systematics (see Ref. [25] for the details). In the following, we will be using only the best fit values for $\chi$ (see also Ref. [23] for an estimation of $\chi$ for Abelian Higgs strings). Another

\footnotetext{
${ }^{1}$ The same parameter $\chi$ enters in the shape of CMB trispectrum generated by cosmic strings [51].
} 


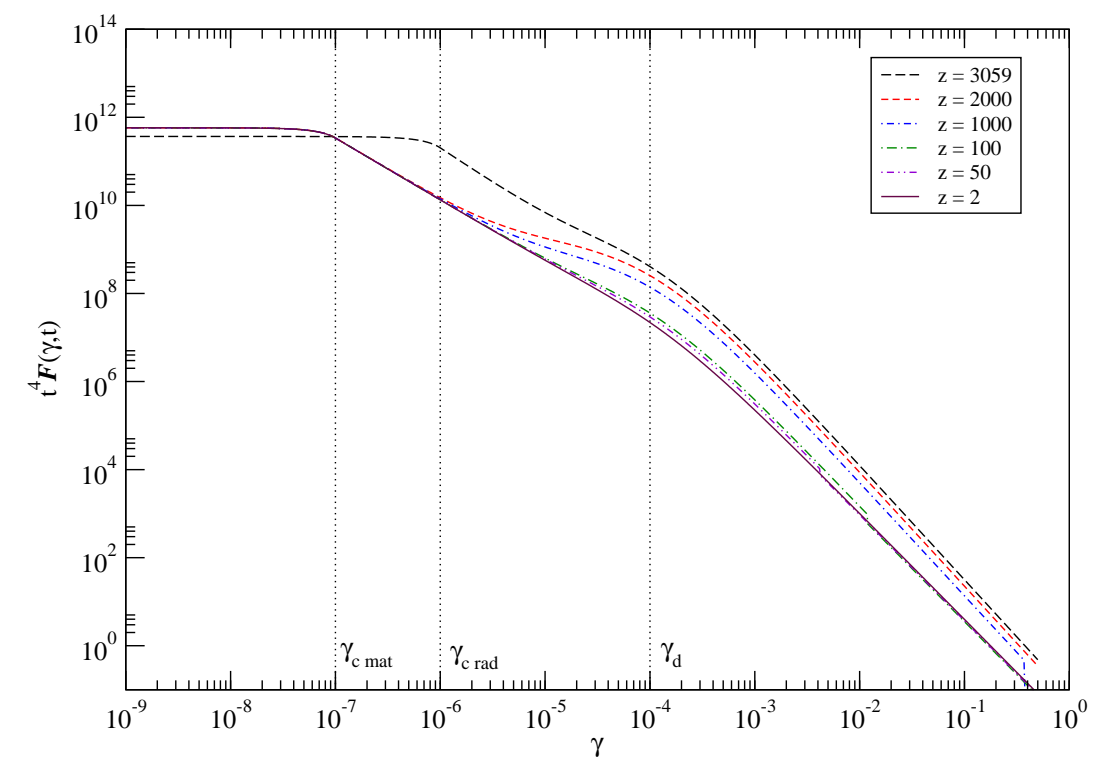

Figure 1. Relaxation of the loop distribution $t^{4} \mathcal{F}(\gamma, z)$ from its scaling shape in the radiation era (black dashed line at redshift $z=3059$ ) towards the matter era attractor (solid line at redshift $z=2$ ). The values for $\gamma_{\mathrm{c}}$ in the matter and radiation era have been arbitrarily chosen for illustration purposes.

advantage of solving a Boltzmann equation for the loop distribution concerns the transition from the radiation era to the matter era as we are able to include any relaxation effects on the loop distribution. The loop distribution at various redshifts around the transition has been represented in figure 1. For completeness, we have included the thermal history effects on the expansion rate of the Universe as these ones have been shown to affect the spectrum at high frequencies [32]. For this purpose, we have used the effective number of relativistic degrees of freedom derived in Ref. [57] while considering that the loop scaling parameters remain unaffected (which is a reasonable assumption).

Concerning the uncertainties associated with the number of kinks and cusps present on the loops, we propose various well motivated scenarios that may be viewed as the remaining theoretical errors on the spectrum. We discuss scenarios having a smooth microstructure and only two cusps, others having a number of kinks ranging from zero to $10^{2}$. For the latter, we show that a new source of gravitational waves on the string could dominate the spectrum: the collisions of left-moving and right-moving kinks. Although the collision amplitude decays faster with frequency than cusps and kinks, it scales as $\omega^{-2}$, kink collisions emit gravitational waves in all directions and the number of event per loop oscillations increases as the square of the number of kinks. In all these scenarios, the number of cusps, kinks and collisions is bounded from above as the total power emitted can never exceed $P_{\mathrm{gw}}=\Gamma G U^{2}$. As a result, one can completely explore the range of the remaining theoretical uncertainties.

The paper is organized as follows. In section 2 we briefly introduce our notation before deriving the main equations for computing the stochastic gravitational wave spectrum. We do not give excessive details on GW emitted by one cusp and one kink as we follow in all points the Damour and Vilenkin calculations [43, 58]. However, some intermediate results have been provided for kink collisions since, up to our knowledge, they have not been considered before for Nambu-Goto strings. Their importance has however been discussed for superstrings 
having Y-junctions in Refs. $[59,60]$. In section 3, we present our results, namely the stochastic gravitational wave spectra expected when loops contain either one cusp, or one kink, or two kinks plus one collision per oscillation. Our findings are critically compared to previous results and when we do not include the above-mentioned new effects, our prediction are in very good agreement with the one of Ref. [37] for $G U=10^{-7}$. For lower values of $G U$, small differences in the power law exponents of the loop distribution, as well as relaxation effects encoded only within our Boltzmann approach, start to play a role and this agreement is accordingly degraded. Physical explanations of the new features are provided along with simple analytic estimates. Finally, in section 4, we explore various motivated models for the string microstructure and estimate how the string tension $G U$ is constrained by the current Laser Interferometer Gravitational-Wave Observatory (LIGO) and European Pulsar Timing Array (EPTA) observations.

\section{Gravitational wave sources}

\subsection{Basic assumptions}

The loops we are interested in are of sub-Hubble sizes such that one can assume their dynamics over one oscillation to be approximately described by the Nambu-Goto equations of motion in Minkowski spacetime. Indeed, as can be seen in figure 1, the distribution vanishes on Hubble length scales, i.e., for $\gamma \geq \gamma_{\infty}$. Let us stress that, in addition to the loops discussed here, a scaling network of cosmic strings exhibit a few large loops with sizes typical of the long strings correlation length, referred to as Kibble loops (see Ref. [11]). These loops are in all points similar to the infinite strings, and so is their contribution to the stochastic gravitational waves background [19]. For convenience, we will take $\gamma_{\infty}=1$ in the following, both in the matter and radiation eras. As discussed in Ref. [43], the size of the loops allows a length scale separation in the derivation of the GW signal. In a local wave zone around each loop, GW emission can be estimated by linearising the metric around a Minkowski background $g_{\mu \nu}=\eta_{\mu \nu}+h_{\mu \nu}$. The emitted GWs then propagate from the local wave zone to the observer along light-like geodesics of an assumed flat Friedman-Lemaitre-Robertson-Walker (FLRW) metric

$$
\mathrm{d} s^{2}=a^{2}(\eta)\left(-\mathrm{d} \eta^{2}+\mathrm{d} \chi^{2}+\chi^{2} \mathrm{~d} \Omega^{2}\right) .
$$

Here $\eta$ and $\chi$ are the conformal time and conformal radius of a spherical coordinate system centered on the source, and $a(\eta)$ is the scale factor. For wavelengths much smaller than the Hubble radius, in addition to frequency redshifting, one finds that the amplitude of $a(\eta) \chi h_{\mu \nu}(\eta, \chi)$ is conserved during propagation thereby allowing to relate the observed GW strain to the one derived around each loop [43].

In practice, defining

$$
\bar{h}_{\mu \nu} \equiv h_{\mu \nu}-\frac{1}{2} h \eta_{\mu \nu}, \quad h \equiv h_{\mu}^{\mu},
$$

supplemented with the harmonic gauge condition $\bar{h}_{, \nu}^{\mu \nu}=0$, the linearised Einstein equations are solved by a retarded propagator and one gets

$$
\bar{h}_{\mathrm{b}}^{\mu \nu}(r)=4 G \int \mathrm{d} t^{\prime} \mathrm{d}^{3} \boldsymbol{r}^{\prime} \frac{T^{\mu \nu}\left(r^{\prime}\right)}{\left|\boldsymbol{r}-\boldsymbol{r}^{\prime}\right|} \delta\left(t-t^{\prime}-\left|\boldsymbol{r}-\boldsymbol{r}^{\prime}\right|\right),
$$

where $G$ is the Newton constant. In this expression $r$ stands for the four-vector at which one evaluates the GW strain while $t$ and $\boldsymbol{r}$ denotes its time and spatial components, respectively. 
The index "b" is a reminder that the result holds only in the local wave zone assuming Minkowski background. Both the stess tensor $T^{\mu \nu}$ and the equations of motion for a cosmic string loop can be obtained from the Nambu-Goto action

$$
S=-U \int \mathrm{d} \tau \mathrm{d} \sigma \sqrt{(\dot{X} \dot{X})^{2}-\dot{X}^{2} \dot{X}^{2}} .
$$

The quantities $X^{\mu}(\tau, \sigma)$ are the embedding functions of the string worlsheet, $\tau$ and $\sigma$ stand for the time-like and space-like internal coordinates, $\dot{X}^{\mu} \equiv \partial X^{\mu} / \partial \sigma$ and $\dot{X}^{\mu} \equiv \partial X^{\mu} / \partial \tau$ (the square root is the determinant of the induced metric along the string worldsheet). From Eq. (2.4), in the temporal and transverse gauge where $X^{0}=\tau=\eta$ and $\dot{\boldsymbol{X}} \cdot \dot{\boldsymbol{X}}=0$, the stress tensor reads

$$
T^{\mu \nu}(r)=U \int \mathrm{d} \sigma\left(-\dot{X}^{\mu} \dot{X}^{\nu}+\dot{X}^{\mu} \dot{X}^{\nu}\right) \delta[\boldsymbol{r}-\boldsymbol{X}(\tau, \sigma)],
$$

while the string dynamics is given by the propagation of left and right moving string deformations [61]

$$
\boldsymbol{X}(\tau, \sigma)=\frac{1}{2}\left[\boldsymbol{X}_{+}\left(\sigma_{+}\right)+\boldsymbol{X}_{-}\left(\sigma_{-}\right)\right]
$$

The vectors $\boldsymbol{X}_{ \pm}$are constant along the characteristics $\sigma_{ \pm} \equiv \sigma \pm \tau$ and verify $\left|\dot{\boldsymbol{X}}_{ \pm}\right|^{2}=1$. By definition of a loop, periodicity conditions in its rest frame require $\boldsymbol{X}(\tau, \sigma+\ell)=\boldsymbol{X}(\tau, \sigma)$ such that $\boldsymbol{X}_{ \pm}\left(\sigma_{ \pm}+\ell\right)=\boldsymbol{X}_{ \pm}\left(\sigma_{ \pm}\right)$are also of period $\ell$. From Eq. (2.6) together with Lorentz invariance along the worldsheet imply that loops oscillate with a period $T=\ell / 2$ as $\boldsymbol{X}(\tau+$ $\ell / 2, \sigma+\ell / 2)=\boldsymbol{X}(\tau, \sigma)$. As a result, in Fourier space, one has

$$
T^{\mu \nu}\left(\varpi_{n}, \boldsymbol{\kappa}\right)=\frac{1}{T} \int \mathrm{d}^{3} \boldsymbol{x} \int_{0}^{T} \mathrm{~d} t T^{\mu \nu}(x) e^{i \varpi_{n} t-i \boldsymbol{\kappa} \cdot \boldsymbol{x}},
$$

where $n$ is an integer and

$$
\varpi_{n}=\frac{2 \pi n}{T}=\frac{4 \pi n}{\ell} .
$$

Plugging Eqs. (2.5) and (2.6) into Eq. (2.7) allows us to obtain an explicit expression for the time domain Fourier transform of the GW tensor in Eq. (2.3). After some algebra, one gets [43]

$$
\bar{h}_{b}^{\mu \nu}\left(\varpi_{n},|\boldsymbol{r}| \hat{\boldsymbol{n}}\right)=\frac{G U}{T} \frac{e^{i \varpi_{n}|\boldsymbol{r}|}}{|\boldsymbol{r}|} C^{\mu \nu}, \quad C^{\mu \nu} \equiv I_{+}^{\mu} I_{-}^{\nu}+I_{+}^{\nu} I_{-}^{\mu},
$$

where quadratic terms $\mathcal{O}\left(1 /|\boldsymbol{r}|^{2}\right)$ have been neglected far from the source. In this expression, the integrals $I_{\epsilon}^{\mu}$ (with $\epsilon= \pm$ ) stand for

$$
I_{\epsilon}^{\mu}\left(\varpi_{n}, \boldsymbol{\kappa}\right) \equiv \int \mathrm{d} \sigma_{\epsilon} \exp \left(\frac{i \varpi_{n} \sigma_{\epsilon}}{2}-\frac{i \boldsymbol{\kappa} \cdot \boldsymbol{X}_{\epsilon}}{2}\right) \frac{\mathrm{d} X_{\epsilon}^{\mu}}{\mathrm{d} \sigma_{\epsilon}}
$$

and should be evaluated on the light cone, namely for $\boldsymbol{\kappa}=\varpi_{n} \hat{\boldsymbol{n}}, \hat{\boldsymbol{n}}$ being an unit vector in the direction of propagation, i.e. $\kappa_{n} \equiv\left(\varpi_{n}, \varpi_{n} \hat{\boldsymbol{n}}\right)$ is light-like. Notice that $X_{\epsilon} \equiv\left(\sigma_{\epsilon}, \boldsymbol{X}_{\epsilon}\right)$ and its derivative $\dot{X}_{\epsilon}^{\mu} \equiv \mathrm{d} X_{\epsilon}^{\mu} / \mathrm{d} \sigma_{\epsilon}$ is also light-like.

Embedding the local wave zone into FLRW spacetime, one can identify $r=a_{\mathrm{em}} \chi, \chi$ being the comoving coordinate of a FLRW coordinate system centered on the source and $a_{\mathrm{em}}$ the scale factor at the time of emission. Including redshifting and using the conservation of $a \chi h^{\mu \nu}$ during propagation, one finally gets for the observed GW strain

$$
\bar{h}^{\mu \nu}\left(\eta_{\mathrm{obs}}, \chi_{\mathrm{obs}}\right)=\frac{G U}{T \chi_{\mathrm{obs}}} \sum_{n=-\infty}^{+\infty} e^{i a_{\mathrm{em}} \varpi_{n}\left(\chi_{\mathrm{obs}}-\eta_{\mathrm{obs}}\right)} C^{\mu \nu}\left(\kappa_{n}\right) .
$$


Notice that in a coordinate system centered on the observer, $\chi_{\mathrm{obs}}$ is also the comoving distance to the source $\chi_{\mathrm{obs}}=\chi_{\mathrm{em}}$

\subsection{Cusps, kinks and collisions}

The amplitude tensor $C^{\mu \nu}$ being the product of rapidly oscillating integrals $I_{\epsilon}^{\mu}$, one can qualitatively infer the large frequency behaviour of the GW strain. From Eq. (2.10), by the Riemann-Lebesgue lemma, the $I_{\epsilon}^{\mu}$ are expected to always decay exponentially fast with $\varpi_{n}$ unless the phase $\varphi=\varpi_{n} \sigma_{\epsilon}-\boldsymbol{\kappa} \cdot \boldsymbol{X}_{\epsilon}$ has a saddle point, and/or the function $\dot{X}_{\epsilon}^{\mu}$ is not smooth.

Saddle points for $I_{\epsilon}^{\mu}\left(\kappa_{n}\right)$ occur when $\hat{\boldsymbol{n}} \cdot \dot{\boldsymbol{X}}_{\epsilon}=1$, i.e. when $\dot{\boldsymbol{X}}_{\epsilon}$ is aligned with the direction of propagation $\hat{\boldsymbol{n}}$. Requiring both $I_{+}^{\mu}$ and $I_{-}^{\nu}$ to have a saddle point therefore implies that $\hat{\boldsymbol{n}}=\dot{\boldsymbol{X}}_{+}=\dot{\boldsymbol{X}}_{-}$, the last equality being the condition for a cosmic string loop to form a "cusp". In terms of worldsheet coordinates, denoting by $\sigma_{\epsilon}^{(\mathrm{s})}$ the values at which the vectors $\dot{\boldsymbol{X}}_{\epsilon}$ coincide with $\hat{\boldsymbol{n}}$, the cusp appears at a specific instant and location given by $\tau^{(\mathrm{s})}=\left(\sigma_{+}^{(\mathrm{s})}-\sigma_{-}^{(\mathrm{s})}\right) / 2$ and $\sigma^{(\mathrm{s})}=\left(\sigma_{+}^{(\mathrm{s})}+\sigma_{-}^{(\mathrm{s})}\right) / 2$. At this point, $\dot{\boldsymbol{X}}^{2}=1$ and the cusp emits a beamed GW burst along the direction $\dot{\boldsymbol{X}}_{+}=\dot{\boldsymbol{X}}_{-}$. The beam opening angle has been derived in Ref. [43] and reads

$$
\theta_{\text {beam }}=\left(\frac{8 \pi}{\sqrt{3} \varpi \ell}\right)^{1 / 3},
$$

where we have omitted the subscript $n$ for $\varpi$ to indicate that the frequency can be considered almost continuous in the large $n$ limit we are interested in.

Discontinuities in the functions $\dot{X}_{\epsilon}^{\mu}\left(\sigma_{\epsilon}\right)$ are common features for cosmic strings and correspond to kinks in the shape of the string. As a result, another configuration for which $C^{\mu \nu}$ does not decrease exponentially fast with frequencies is when one of the integral $I_{\epsilon}^{\mu}$ develops a saddle point at $\sigma_{\epsilon}^{(\mathrm{s})}$ and the other $I_{-\epsilon}^{\nu}$ a kink at $\sigma_{-\epsilon}^{(\mathrm{k})}$. From Eq. (2.6), the string worldsheet $\boldsymbol{X}$ has indeed a kink propagating along one direction $\sigma=\sigma_{-\epsilon}^{(\mathrm{k})}+\epsilon \tau$. Along these locations, the saddle point of $I_{\epsilon}^{\mu}$ occurs along the $\dot{\boldsymbol{X}}_{\epsilon}$ direction only such that GW are beamed in a light-house manner by the propagating kink.

Both of these situations have been proposed and exhaustively studied in Ref. [43] and we simply quote the result. Keeping the dominant terms of a Taylor expansion around the saddle point at $\sigma_{\epsilon}=\sigma_{\epsilon}^{(\mathrm{s})}$, one gets for the cusp case

$$
\left.I_{\epsilon}^{\mu}(\kappa)\right|_{\mathrm{c}} \simeq i \frac{\varpi}{|\varpi|}\left(\frac{2}{3}\right)^{1 / 3} \frac{4 \pi}{\Gamma\left(\frac{1}{3}\right)} \frac{\ddot{X}_{\epsilon}^{\mu}\left(\sigma_{\epsilon}^{(\mathrm{s})}\right)}{\left|\ddot{\boldsymbol{X}}_{\epsilon}\left(\sigma_{\epsilon}^{(\mathrm{s})}\right)\right|^{4 / 3}|\varpi|^{2 / 3}} .
$$

From dimensional arguments, one can take $\left|\ddot{\boldsymbol{X}}_{\epsilon}\right|=2 \pi \beta / \ell$ with $\beta=\mathcal{O}(1)$ and the overall numerical factor

$$
\eta_{\mathrm{s}} \equiv\left(\frac{2}{3}\right)^{1 / 3} \frac{4 \pi}{\Gamma\left(\frac{1}{3}\right)},
$$

is approximately $\eta_{\mathrm{s}} \simeq 4$.1. For kinks, one can define the discontinuity amplitude vectors $u_{\epsilon 1}^{\mu}$ and $u_{\epsilon 2}^{\mu}$ such that

$$
\lim _{\sigma_{\epsilon} \rightarrow \sigma_{\epsilon}^{(\mathrm{k})}-0} \dot{X}_{\epsilon}^{\mu}\left(\sigma_{\epsilon}\right)=u_{\epsilon 1}^{\mu}, \quad \lim _{\sigma_{\epsilon} \rightarrow \sigma_{\epsilon}^{(\mathrm{k})}+0} \dot{X}_{\epsilon}^{\mu}\left(\sigma_{\epsilon}\right)=u_{\epsilon 2}^{\mu}
$$


Both $u_{\epsilon 1}$ and $u_{\epsilon 2}$ are null vectors. At leading order, the integrals (2.10) read

$$
\left.I_{\epsilon}^{\mu}(\kappa)\right|_{\mathrm{k}} \simeq 2 i\left(\frac{u_{\epsilon 1}^{\mu}}{\kappa \cdot u_{\epsilon 1}}-\frac{u_{\epsilon 2}^{\mu}}{\kappa \cdot u_{\epsilon 2}}\right)=\frac{2 i}{\varpi} v_{\epsilon}^{\mu},
$$

where we have defined

$$
v_{\epsilon}^{\mu} \equiv \frac{u_{\epsilon 1}^{\mu}}{\hat{\kappa} \cdot u_{\epsilon 1}}-\frac{u_{\epsilon 2}^{\mu}}{\hat{\kappa} \cdot u_{\epsilon 2}}, \quad \hat{\kappa} \equiv(1, \hat{\boldsymbol{n}}) .
$$

From Eqs. (2.13) and (2.16), one concludes that, at high frequencies, $C^{\mu \nu} \propto \varpi^{-4 / 3}$ for cusps and $C^{\mu \nu} \propto \varpi^{-5 / 3}$ for kinks. As a result, if cusps develop on a loop, they should dominate GW emission [43].

However, such a conclusion may not hold if the number of kinks propagating on a cosmic string loop is large, a situation which may occur even in presence of GW backreaction [46, 47]. As a matter of fact, kinks themselves render the appearance of cusps less likely [45]. Moreover, because kinks are formed during string intersections, they are created in pairs and each kinky loop is expected to have an equal number of left- and right-moving kinks. This suggests that, in addition to the two previously discussed situations, another significant source of GW emission occurs when both integrals $I_{\epsilon}^{\mu}$ and $I_{-\epsilon}^{\nu}$ behave as in Eq. (2.16), which implies that $C^{\mu \nu} \propto \varpi^{-2}$ at large frequencies. Fixing both $\sigma_{+}=\sigma_{+}^{(\mathrm{k})}$ and $\sigma_{-}=\sigma_{-}^{(\mathrm{k})}$ corresponds to an unique event along the worldsheet, at $\tau^{(\mathrm{k})}=\left(\sigma_{+}^{(\mathrm{k})}-\sigma_{-}^{(\mathrm{k})}\right) / 2$ and $\sigma^{(\mathrm{k})}=\left(\sigma_{+}^{(\mathrm{k})}+\sigma_{-}^{(\mathrm{k})}\right) / 2$, which describes the collision of two kinks moving in opposite directions. The GW emission does not depend on $\hat{\boldsymbol{n}}$ and is therefore isotropic. Although $C^{\mu \nu}$ is decreasing faster with frequencies than in the cusp and kink case, one should keep in mind that the number of kink collisions per loop oscillation scales as the square of the number of kinks. Indeed, if a loop exhibits $N_{\mathrm{k}}$ kinks, in an equal number of left-movers and right-movers, the number of collisions per loop oscillation is $N_{\mathrm{k}}^{2} / 4$. As we show later on, kinks collision may actually dominate the overall GW signal for very kinky loops.

\subsection{Single loop energy and spectral density}

From the pseudo stress tensor associated with $h_{\mu \nu}$, one can estimate the mean energy density associated with the waveform of Eq. (2.11) at the observer's location. Taking the scale factor today $a_{0}=1$ and averaging over the observed loop oscillation period, i.e. $T / a_{\mathrm{em}}$, one gets

$$
\rho_{\mathrm{gw}}^{(\circ)}=\frac{1}{32 \pi G} \frac{a_{\mathrm{em}}}{T} \int_{0}^{T / a_{\mathrm{em}}}\left(\dot{\overline{\bar{h}}}_{\alpha \beta} \dot{\bar{h}}^{\alpha \beta}-\frac{1}{2} \dot{\bar{h}}^{2}\right) \mathrm{d} \eta,
$$

where a dot denotes derivative with respect to the conformal time. Plugging Eq. (2.11) into this expression yields

$$
\rho_{\mathrm{gw}}^{(\circ)}=\frac{1}{16 \pi G}\left(\frac{G U}{T \chi_{\mathrm{em}}}\right)^{2} \sum_{n=1}^{+\infty}\left(a_{\mathrm{em}} \varpi_{n}\right)^{2}\left[C_{\alpha \beta}^{*}\left(\kappa_{n}\right) C^{\alpha \beta}\left(\kappa_{n}\right)-\frac{1}{2}\left|C\left(\kappa_{n}\right)\right|^{2}\right] .
$$

Each angular frequency $\varpi_{n}$ is redshifted and observed as $\omega_{n} \equiv a_{\mathrm{em}} \varpi_{n}$. Moreover, Eq. (2.19) can be further reduced by taking the continuum limit over $\omega_{n}$

$$
\rho_{\mathrm{gw}}^{(\circ)}=\frac{T}{32 \pi^{2} G a_{\mathrm{em}}}\left(\frac{G U}{T \chi_{\mathrm{em}}}\right)^{2} \int_{\omega_{1}}^{+\infty} \mathrm{d} \omega \omega^{2}\left[C_{\alpha \beta}^{*}\left(\frac{k}{a_{\mathrm{em}}}\right) C^{\alpha \beta}\left(\frac{k}{a_{\mathrm{em}}}\right)-\frac{1}{2}\left|C\left(\frac{k}{a_{\mathrm{em}}}\right)\right|^{2}\right],
$$


where the four-vector $k \equiv(\omega, \omega \hat{\boldsymbol{n}})$. Notice that the integral has a lower cut-off in frequencies which corresponds to the fundamental mode $n=1$, i.e.

$$
\omega_{1}\left(\ell, z_{\mathrm{em}}\right)=\frac{2 \pi a_{\mathrm{em}}}{T}=\frac{4 \pi}{\left(1+z_{\mathrm{em}}\right) \ell} .
$$

We define the spectral density parameter of GW today, $\Omega_{\mathrm{gw}}(\omega)$, by

$$
\frac{\rho_{\mathrm{gw}}}{\rho_{\text {crit }}} \equiv \int_{0}^{+\infty} \frac{\mathrm{d} \omega}{\omega} \Omega_{\mathrm{gw}}(\omega),
$$

where the critical density is $\rho_{\text {crit }}=3 H_{0}^{2} /(8 \pi G), H_{0}$ being the Hubble parameter today. Comparing this expression to Eq. (2.20), we obtain for a single loop, assuming the observer to be within the beam

$$
\Omega_{\mathrm{gw}}^{(\circ)}(\omega)=\frac{(G U)^{2}}{12 \pi H_{0}^{2}} \frac{\omega^{3}}{T a_{\mathrm{em}} \chi_{\mathrm{em}}^{2}}\left[C_{\alpha \beta}^{*}\left(\frac{k}{a_{\mathrm{em}}}\right) C^{\alpha \beta}\left(\frac{k}{a_{\mathrm{em}}}\right)-\frac{1}{2}\left|C\left(\frac{k}{a_{\mathrm{em}}}\right)\right|^{2}\right] \Theta\left(\omega-\omega_{1}\right) .
$$

In order to estimate the observed stochastic GW spectrum, one has still to define which events are stochastic from the observer point of view and add up the contribution of all loops in the Universe.

\section{Stochastic gravitational wave spectrum}

From the cosmological loop distribution $\mathcal{F}(\ell, z)$, the number of loops of size $\ell$ at redshift $z$ per $\mathrm{d} \ell \mathrm{d} z$ volume in a flat FLRW metric reads

$$
\frac{\mathrm{d} N_{\circ}}{\mathrm{d} \ell \mathrm{d} z}=\frac{\mathrm{d} V}{\mathrm{~d} z} \mathcal{F}(\ell, z), \quad \frac{\mathrm{d} V}{\mathrm{~d} z}=\frac{4 \pi \chi^{2}(z)}{(1+z)^{3} H(z)},
$$

where $H(z)$ stands for the Hubble parameter at redshift $z$. Assuming for the time being that all of these loops have only one GW emission event per oscillation, either a cusp, a kink or a collision, one can estimate their contribution to the GW event rate today, at angular frequency $\omega$, by

$$
\frac{\mathrm{d} N_{\mathrm{gw}}}{\mathrm{d} z \mathrm{~d} \ell \mathrm{d} \eta}=\frac{2}{(1+z) \ell} \frac{\mathrm{d} V}{\mathrm{~d} z} \mathcal{F}(\ell, z) \Theta\left[\omega-\omega_{1}(\ell, z)\right] \Delta_{\text {beam }}(\omega, \ell, z),
$$

where the time averaging is performed over one oscillation period today, i.e. for $\Delta \eta_{0}=$ $(1+z) T$. This only makes sense for frequencies higher than the redshifted fundamental mode $\omega_{1}$, as it was the case for Eq. (2.20). The beam factor $\Delta_{\text {beam }}(\omega, \ell, z)=\Omega_{\text {beam }} /(4 \pi)$ is the probability for the observer to be within the GW emission solid angle $\Omega_{\text {beam }}$. From Eq. (2.12), it reads

$$
\begin{aligned}
& \left.\Delta_{\text {beam }}(\omega, \ell, z)\right|_{\mathrm{c}}=\frac{\pi \theta_{\text {beam }}^{2}}{4 \pi}=\frac{1}{4}\left[\frac{8 \pi}{\sqrt{3} \omega(1+z) \ell}\right]^{2 / 3}, \\
& \left.\Delta_{\text {beam }}(\omega, \ell, z)\right|_{\mathrm{k}}=\frac{2 \pi \theta_{\text {beam }}}{4 \pi}=\frac{1}{2}\left[\frac{8 \pi}{\sqrt{3} \omega(1+z) \ell}\right]^{1 / 3},
\end{aligned}
$$

for cusp and kink, respectively, while $\left.\Delta_{\text {beam }}(\omega, \ell, z)\right|_{\mathrm{kk}}=1$ for kinks collision. 
Even for a perfect GW detector with infinite sensitivity, all of these events superimpose and may not be detected individually, especially if their rate of occurrence exceeds the detector frequency, here assumed to be $f=\omega /(2 \pi)$. As pointed in Ref. [43], all individually separable events will be observed as bursts and cannot be counted within the stochastic background. In order to separate the burst-like events from the stochastic ones, we have followed the method of Ref. [62, 63]. It assumes that the event rate is a monotonic decreasing function of the GW strain power such that the separation between burst-like and stochastic-like event can be made on the values of $\left|h_{\mu \nu}(\omega)\right|^{2}$. Because we do not attempt to disambiguate the polarization degrees of freedom, let us define

$$
\bar{h}_{\mathrm{c}}^{2}\left(\eta_{\mathrm{obs}}, \chi_{\mathrm{obs}}\right) \equiv\left\langle\bar{h}_{\mu \nu}^{*} \bar{h}^{\mu \nu}\right\rangle-\frac{1}{2}\left\langle|\bar{h}|^{2}\right\rangle,
$$

where $\langle A\rangle$ is average of quantity $A$ over a redshifted oscillation period. From Eq. (2.11), averaging over a redshifted oscillation period $\Delta \eta_{0}$ and taking the continuum limit in $\varpi_{n}$, one gets per event:

$$
\bar{h}_{\mathrm{c}}^{2}\left(\eta_{\mathrm{obs}}, \chi_{\mathrm{obs}}\right)=\frac{2 T}{a_{\mathrm{em}}}\left(\frac{G U}{T \chi_{\mathrm{obs}}}\right)^{2} \int_{\omega_{1}}^{+\infty} \frac{\mathrm{d} \omega}{2 \pi}\left[C_{\alpha \beta}^{*}\left(\frac{k}{a_{\mathrm{em}}}\right) C^{\alpha \beta}\left(\frac{k}{a_{\mathrm{em}}}\right)-\frac{1}{2}\left|C\left(\frac{k}{a_{\mathrm{em}}}\right)\right|^{2}\right] .
$$

The GW strain power at frequency $\omega$ per event is thus given by

$$
\bar{h}_{\mathrm{c}}^{2}(\omega, \ell, z)=\left[\frac{G U(1+z)}{\chi(z)}\right]^{2} \overline{\mathcal{C}}^{2}[k(1+z)] \Theta\left[\omega-\omega_{1}(\ell, z)\right]
$$

where we have defined

$$
\overline{\mathcal{C}}^{2} \equiv C_{\alpha \beta}^{*} C^{\alpha \beta}-\frac{1}{2}|C|^{2} .
$$

From Eq. (3.2), the minimal amplitude $\bar{h}_{\star}(\omega)$ under which burst-like events will not be time separable any more is solution of

$$
\iint \mathrm{d} z \mathrm{~d} \ell \frac{2}{(1+z) \ell} \frac{\mathrm{d} V}{\mathrm{~d} z} \mathcal{F}(\ell, z) \Delta_{\text {beam }}(\omega, \ell, z) \Theta\left[\omega-\omega_{1}(\ell, z)\right] \Theta\left[\bar{h}_{\mathrm{c}}(\omega, \ell, z)-\bar{h}_{\star}(\omega)\right]=\frac{\omega}{2 \pi} .
$$

From Eqs. (2.23), (3.2), (3.6) and (3.8), one obtains for the total stochastic gravitational wave spectrum

$$
\begin{aligned}
\Omega_{\mathrm{sgw}}(\omega) & =\frac{(G U)^{2} \omega^{3}}{6 \pi H_{0}^{2}} \iint \mathrm{d} z \mathrm{~d} \ell \frac{\mathrm{d} V}{\mathrm{~d} z} \frac{\mathcal{F}(\ell, z)}{\ell(1+z)} \Delta_{\text {beam }}(\omega, \ell, z) \\
& \times \frac{(1+z)^{2}}{\chi^{2}(z)} \overline{\mathcal{C}}^{2}(\omega, \ell, z) \Theta\left[\omega-\omega_{1}(\ell, z)\right] \Theta\left[\overline{\mathcal{C}}_{\star}(\omega, z)-\overline{\mathcal{C}}(\omega, \ell, z)\right],
\end{aligned}
$$

where the rescaled stochastic threshold $\overline{\mathcal{C}}_{\star}$ stands for

$$
\overline{\mathcal{C}}_{\star}(\omega, z) \equiv \frac{\chi(z)}{(1+z) G U} \bar{h}_{\star}(\omega) .
$$

Notice that the previous expressions are valid assuming only one cusp, or one kink, or one collision event per loop oscillation. The generalization to multiple events of one kind is however trivial as it is enough to rescale, for instance, $\mathcal{F}$ by the number of events per loop oscillation in all the previous formulas. 


\subsection{Unified source functions and scaling variables}

As discussed in the introduction, up to the transition from radiation to matter and the latetime acceleration of the Universe, the loop scaling distribution is stationary in terms of the variable $\gamma=\ell / t$, where $t$ is the cosmic time. It is therefore convenient to change variables from $(\ell, z)$ to $(\gamma, z)$ in Eq. (3.9). After expanding the various cosmological factors, one gets

$$
\begin{aligned}
\Omega_{\mathrm{sgw}}(\omega) & =\frac{2(G U)^{2} \omega^{3}}{3 H_{0}^{3}} \iint \mathrm{d} z \mathrm{~d} \gamma \frac{t^{4}(z) \mathcal{F}(\gamma, z)}{\gamma(1+z)^{2}} \frac{H_{0}}{H(z)} \Delta_{\text {beam }}(\omega, \gamma, z) \\
& \times \tilde{\mathcal{C}}^{2}(\omega, \gamma, z) \Theta\left[\omega-\frac{4 \pi}{t(z)(1+z) \gamma}\right] \Theta\left[\tilde{\mathcal{C}}_{\star}(\omega, z)-\tilde{\mathcal{C}}(\omega, \gamma, z)\right],
\end{aligned}
$$

in which we have rendered explicit the stationary scaling distribution $t^{4} \mathcal{F}$. We have also introduced dimensionless quantities such as $\tilde{\mathcal{C}}^{2} \equiv \overline{\mathcal{C}}^{2} / t^{4}(z)$. Accordingly, one can define the dimensionless stochastic threshold

$$
\tilde{h}_{\star}(\omega) \equiv \frac{H_{0}}{G U} \bar{h}_{\star}(\omega)
$$

such that

$$
\tilde{\mathcal{C}}_{\star}(\omega, z)=\frac{\chi(z)}{H_{0} t^{2}(z)(1+z)} \tilde{h}_{\star}(\omega),
$$

is also dimensionless. From Eq. (3.8), in terms of $\gamma$ and $z, \tilde{h}_{\star}(\omega)$ is obtained by solving the rate equation

$$
\begin{aligned}
& \iint \mathrm{d} z \mathrm{~d} \gamma \frac{t^{4}(z) \mathcal{F}(\gamma, z)}{\gamma(1+z)^{4}} \frac{H_{0}}{H(z)} \frac{\chi^{2}(z)}{t^{2}(z)} \frac{8 \pi \Delta_{\text {beam }}(\omega, \gamma, z)}{t^{2}(z) H_{0}^{2}} \\
& \times \Theta\left[\omega-\frac{4 \pi}{t(z)(1+z) \gamma}\right] \Theta\left[\tilde{\mathcal{C}}(\omega, \gamma, z)-\frac{\chi(z)}{H_{0} t^{2}(z)(1+z)} \tilde{h}_{\star}(\omega)\right]=\frac{\omega}{2 \pi H_{0}} .
\end{aligned}
$$

Depending on the GW source type, cusp, kink or collision, both $\Delta_{\text {beam }}$ and $\tilde{\mathcal{C}}$ are changed such that these two equations should be solved separately for each case. However, from Eq. (3.3), one can see that all beam factors are of the form

$$
\Delta_{\text {beam }}(\omega, \gamma, z)=\frac{b_{\alpha}}{\gamma^{1-1 / \alpha}}\left[\frac{8 \pi}{\sqrt{3} \omega t(z)(1+z)}\right]^{1-1 / \alpha},
$$

where the parameter $\alpha=3$ for cusps, $\alpha=3 / 2$ for kinks and $\alpha=1$ for collisions. The coefficient $b_{\alpha}$ is a numerical constant equals to $1 / 4,1 / 2$ or 1 , respectively. Plugging the integrals $I_{\epsilon}^{\mu}$ quoted in Eqs. (2.13) and (2.16) into Eq. (3.7), one gets

$$
\begin{aligned}
\tilde{\mathcal{C}}_{\mathrm{c}}(\omega, \gamma, z) & =\frac{\eta_{\mathrm{s}}^{2} \sqrt{2}}{(2 \pi \beta)^{2 / 3}} \frac{\gamma^{2 / 3}}{[\omega t(z)(1+z)]^{4 / 3}} \\
\tilde{\mathcal{C}}_{\mathrm{k}}(\omega, \gamma, z) & =\frac{2 \eta_{\mathrm{s}} \sqrt{2 v_{ \pm}^{2}}}{(2 \pi \beta)^{1 / 3}} \frac{\gamma^{1 / 3}}{[\omega t(z)(1+z)]^{5 / 3}} \\
\tilde{\mathcal{C}}_{\mathrm{kk}}(\omega, \gamma, z) & =4 \sqrt{2 v_{+}^{2} v_{-}^{2}} \frac{1}{[\omega t(z)(1+z)]^{2}},
\end{aligned}
$$


for the cusp, kink and collision case. Let us notice that $u_{\epsilon 1}, u_{\epsilon 2}$ and $\hat{\kappa}$ in Eq. (2.17) are all future directed null vectors and this ensures that both $v_{+}^{2}$ and $v_{-}^{2}$ are positive. As a result, all the source functions $\tilde{\mathcal{C}}(\omega, \gamma, z)$ can also be unified as

$$
\tilde{\mathcal{C}}(\omega, \gamma, z)=c_{\alpha} \frac{\gamma^{1-1 / \alpha}}{[\omega t(z)(1+z)]^{1+1 / \alpha}} .
$$

The numerical constants $c_{\alpha}$ can be read from Eq. (3.16) and contains all the theoretical uncertainties associated with each of the GW source type

$$
c_{3} \equiv \frac{\eta_{\mathrm{s}}^{2} \sqrt{2}}{(2 \pi \beta)^{2 / 3}}, \quad c_{3 / 2} \equiv \frac{2 \eta_{\mathrm{s}} \sqrt{2 v_{ \pm}^{2}}}{(2 \pi \beta)^{1 / 3}}, \quad c_{1} \equiv 4 \sqrt{2 v_{+}^{2} v_{-}^{2}} .
$$

From Eqs. (3.11) and (3.14), these theoretical uncertainties can be trivially absorbed into our final set of rescaled dimensionless variables defined by

$$
\hat{\mathcal{C}}(\omega, \gamma, z) \equiv \frac{\tilde{\mathcal{C}}(\omega, \gamma, z)}{c_{\alpha}}, \quad \hat{h}_{\star}(\omega) \equiv \frac{\tilde{h}_{\star}(\omega)}{c_{\alpha}}=\frac{H_{0}}{G U c_{\alpha}} \bar{h}_{\star}(\omega), \quad \hat{\Omega}_{\mathrm{sgw}}(\omega) \equiv \frac{\Omega_{\mathrm{sgw}}(\omega)}{c_{\alpha}^{2}}
$$

All "hat" quantities are therefore independent of the value of $\eta_{\mathrm{s}}$ and $\beta$ while the physical quantities, as for instance $\Omega_{\mathrm{sgw}}(\omega)$, can be recovered from the above formulas.

\subsection{Loop visibility domains}

The Heaviside functions in Eqs. (3.11) and (3.14) directly stem from the existence of the minimal frequency $\omega_{1}$ and the requirement of keeping only stochastic-like events. In terms of the variables $(\gamma, z)$, they define some integration domains which physically take into account frequency redshifting as well as the fact that a GW burst-like signal may either come from a very distant high amplitude emission event or a close small amplitude one. Let us define the function $\gamma_{1}(\omega, z)$ by

$$
\gamma_{1}(\omega, z) \equiv \frac{4 \pi}{H_{0} t(z)(1+z)} \frac{H_{0}}{\omega} .
$$

From this definition, the first Heaviside function in both Eqs. (3.11) and (3.14) will be nonvanishing only for $\gamma \geq \gamma_{1}(\omega, z)$. The fundamental mode of each loop therefore imposes a lower cut-off in the size of the loops that are "visible" at the angular frequency $\omega$.

Similarly, we can define, for $\alpha \neq 1$, the length scale

$$
\gamma_{\star}\left(\omega, z, \hat{h}_{\star}\right) \equiv\left[\hat{h}_{\star} \frac{\chi(z)}{t(z)}\right]^{\frac{\alpha}{\alpha-1}}\left[H_{0} t(z)(1+z)\right]^{\frac{1}{\alpha-1}}\left(\frac{\omega}{H_{0}}\right)^{\frac{\alpha+1}{\alpha-1}},
$$

such that events counted in the burst rate only come from the domain $\gamma \geq \gamma_{\star}$, see the second Heaviside function in Eq. (3.14). Conversely, stochastic events appearing in Eq. (3.11) are those verifying $\gamma<\gamma_{\star}$. Kink collision, $\alpha=1$, is a particular case and will be discussed below.

In figure 2 , we have represented both $\gamma_{1}(\omega, z)$ and $\gamma_{\star}\left(\omega, z, \hat{h}_{\star}\right)$ for the kink case $\alpha=3 / 2$ and at frequency $f=1 \mathrm{~Hz}$. The low redshift behaviour of these functions can be obtained by Taylor expanding Eqs. (3.20) and (3.21) and one gets

$$
\gamma_{1}(\omega, z \ll 1) \simeq \frac{4 \pi}{H_{0} t_{0}} \frac{H_{0}}{\omega}, \quad \gamma_{\star}\left(\omega, z \ll 1, \hat{h}_{\star}\right) \simeq \frac{1}{H_{0} t_{0}}\left(\hat{h}_{\star} z\right)^{\frac{\alpha}{\alpha-1}}\left(\frac{\omega}{H_{0}}\right)^{\frac{\alpha+1}{\alpha-1}},
$$




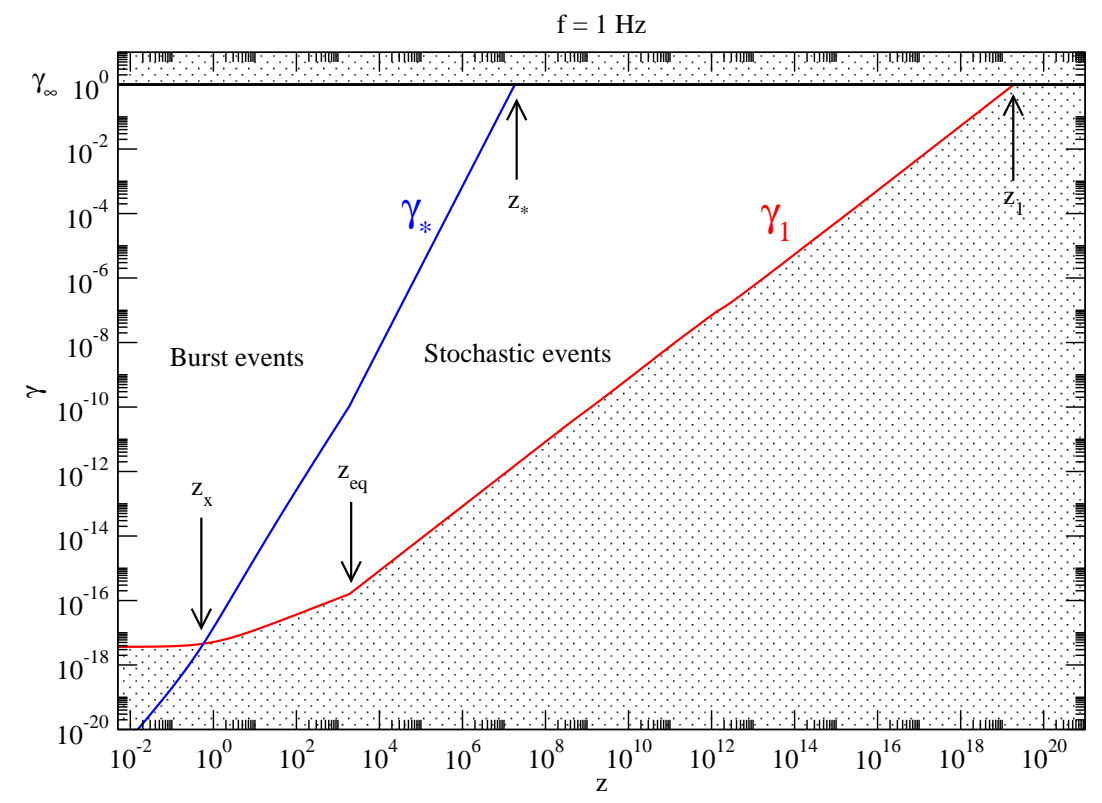

Figure 2. Loop visibility domains for GW kink events observed at frequency $f=1 \mathrm{~Hz}$ in the plane $(\gamma=\ell / t, z)$. Only loops having $\gamma>\gamma_{1}(\omega, z)$ oscillate slow enough to have a redshifted fundamental mode $\omega_{1}<\omega$. The curve $\gamma_{\star}\left(\omega, z, \hat{h}_{\star}\right)$ separates the domain in two regions. For $\gamma<\gamma_{\star}$, the observed GW events occur fast enough to be interpreted as stochastic, whereas they could be individually isolated for all loops having $\gamma>\gamma_{\star}$. For the illustration, we have chosen $\hat{h}_{\star} \simeq 5 \times 10^{-37}$, which solves the rate equation (3.14) for a loop distribution typical of $G U=10^{-7}$.

where $t_{0}$ is the age of the Universe. There is a crossover redshift, $z_{\times}$, such that for $z<z_{\times}$ one has $\gamma_{\star}<\gamma_{1}$ and for $z>z_{\times}, \gamma_{\star}>\gamma_{1}$. This redshift is solution of

$$
H_{0} \chi\left(z_{\times}\right)\left(1+z_{\times}\right)=\frac{(4 \pi)^{\frac{\alpha-1}{\alpha}}}{\hat{h}_{\star}}\left(\frac{H_{0}}{\omega}\right)^{2} .
$$

Notice that this equation is regular for $\alpha=1$ and this allows us to discuss the kink collision case. From the argument of the second Heaviside function in Eq. (3.14), taking the kink collision value for $\tilde{\mathcal{C}}$ in Eq. (3.16), one sees that there is no any explicit dependency in $\gamma$ and the condition for having only burst-like events simplifies to $z<z_{\times}, z_{\times}$being given by the above expression with $\alpha=1$. As a result, the kink collision case can be viewed in figure 2 as the limiting situation in which the slope of the $\gamma_{\star}\left(\omega, z, \hat{h}_{\star}\right)$ function becomes infinite. Independently of the loop distribution, all kink collisions close to the observer, namely occurring at $z<z_{\times}$are well separated GW bursts, as opposed to those occurring at $z>z_{\times}$which are accounted for as stochastic. For cusp events, the shape of $\gamma_{\star}$ is very similar to the one plotted in figure 2 only the slope is steeper while $\gamma_{1}$ remains the same for all GW source types (see figure 3).

At large redshifts, assuming the background to be pure radiation era, one gets

$$
\begin{aligned}
\gamma_{1}\left(\omega, z \gg z_{\mathrm{eq}}\right) & \simeq 8 \pi \sqrt{\Omega_{\mathrm{rad}}} z \frac{H_{0}}{\omega}, \\
\gamma_{\star}\left(\omega, z \gg z_{\mathrm{eq}}, \hat{h}_{\star}\right) & \simeq 2 \sqrt{\Omega_{\mathrm{rad}}}\left(H_{0} \chi_{0} \hat{h}_{\star}\right)^{\frac{\alpha}{\alpha-1}} z^{1+\frac{\alpha}{\alpha-1}}\left(\frac{\omega}{H_{0}}\right)^{\frac{\alpha+1}{\alpha-1}} .
\end{aligned}
$$




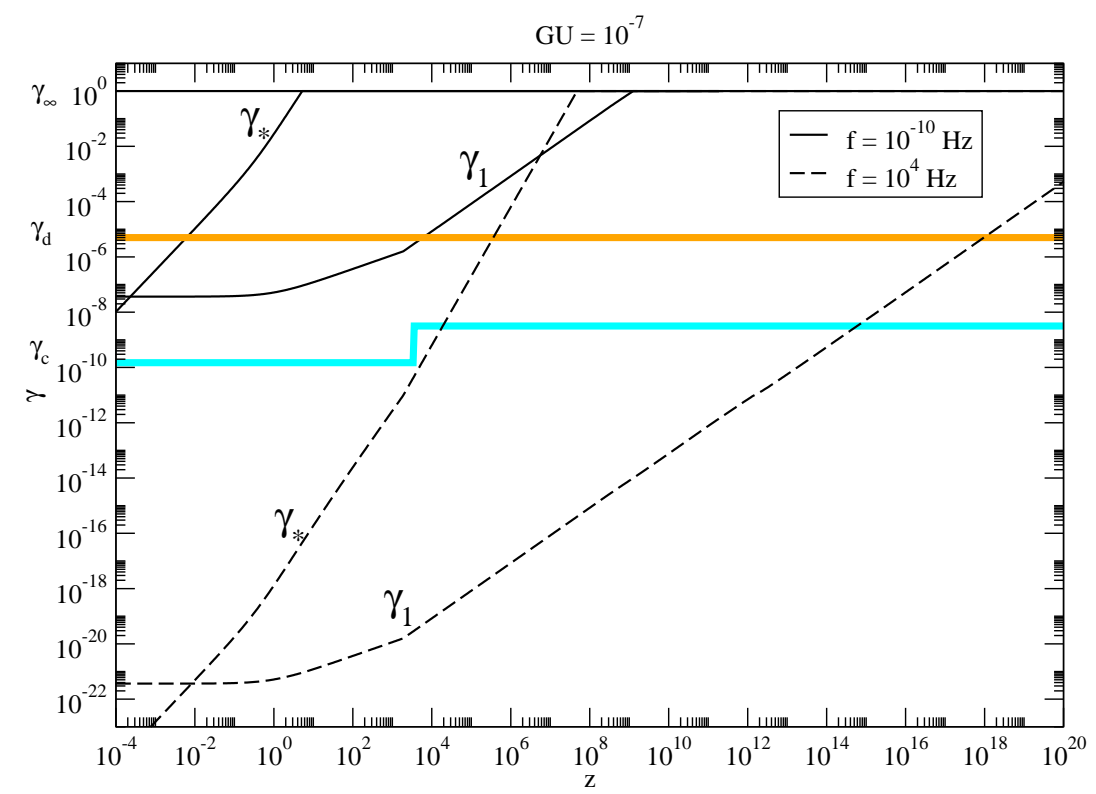

Figure 3. Dependence of the burst and stochastic domains in the plane $(\gamma, z)$ with respect to the observation frequency $f=\omega /(2 \pi)$ (cusp case with $\alpha=3$ ). The solid lines represent the boundary functions $\gamma_{1}(\omega, z)$ and $\gamma_{\star}\left(\omega, z, \hat{h}_{\star}\right)$ for $f=10^{-10} \mathrm{~Hz}$ while the dashed lines are for $f=10^{4} \mathrm{~Hz}$. Values for $\hat{h}_{\star}(\omega)$ have been chosen to solve the rate equation for $G U=10^{-7}$. The two horizontal bands are the gravitational emission scale $\gamma_{\mathrm{d}}$, and backreaction scale $\gamma_{\mathrm{c}}$, for the loop distribution. Depending on $\omega$, they can be in or out the integration domains.

As a result, $\gamma_{\star}$ grows always faster than $\gamma_{1}$ at large redshifts and once $z>z_{\times}$, one always has the hierarchy $\gamma_{\star}\left(\omega, z, \hat{h}_{\star}\right)>\gamma_{1}(\omega, z)$ as in figure 2 . Notice that the loop distribution vanishes on super-horizon distances such that all integrals are also vanishing for $\gamma>\gamma_{\infty}$. It implies that there are two other relevant redshifts, $z_{1}$ and $z_{\star}$, defined by $\gamma_{1}\left(\omega, z_{1}\right)=\gamma_{\infty}$ and $\gamma_{\star}\left(\omega, z_{\star}, \hat{h}_{\star}\right)=\gamma_{\infty}$, respectively. They are the solutions of

$$
H_{0} t\left(z_{1}\right)\left(1+z_{1}\right)=\frac{4 \pi}{\gamma_{\infty}} \frac{H_{0}}{\omega}, \quad\left[\frac{\chi\left(z_{\star}\right)}{t\left(z_{\star}\right)}\right]^{\alpha} H_{0} t\left(z_{\star}\right)\left(1+z_{\star}\right)=\frac{\gamma_{\infty}^{\alpha-1}}{\hat{h}_{\star}^{\alpha}}\left(\frac{H_{0}}{\omega}\right)^{\alpha+1},
$$

and have been represented in figure 2 . The redshift $z_{\star}$ is the frontier above which all GW emission events observed at angular frequency $\omega$ will be classified as stochastic, while $z_{1}$ is the maximum observable redshift at this frequency.

In figure 3, we have represented the dependence of the domain boundaries $\gamma_{1}$ and $\gamma_{\star}$ with respect to the observed frequency $f=\omega /(2 \pi)$ and for the cusp case $(\alpha=3)$. Although the dependence of $\gamma_{1}(\omega, z)$ is explicit in Eq. (3.20), it varies as $\omega^{-1}, \gamma_{\star}\left(\omega, z, \hat{h}_{\star}\right)$ has a nontrivial behaviour due to its dependence on the values of $\hat{h}_{\star}(\omega)$. For the illustration, we have chosen values of $\hat{h}_{\star}(\omega)$ solving the rate equation for a loop distribution with $G U=10^{-7}$. This loop distribution has a gravitational emission scale at $\gamma_{\mathrm{d}} \simeq 5 \times 10^{-6}$, represented by a horizontal orange band in figure 3. The gravitational backreaction scale $\gamma_{\mathrm{c}} \simeq 2 \times 10^{-10}$ in the matter era while $\gamma_{\mathrm{c}} \simeq 3 \times 10^{-9}$ in the radiation era. It is represented as a blue discontinuous band. For most of the cosmological history, the loop distribution is in scaling and assumes a shape as plotted in figure 1, namely $t^{4}(z) \mathcal{F}(\gamma, z)$ is independent of $z$, maximal for $\gamma<\gamma_{\mathrm{c}}$ and decreases for $\gamma>\gamma_{\mathrm{d}}$ to vanish at $\gamma=\gamma_{\infty}$. From this observation, one can conclude 
without any calculation that the stochastic gravitational wave spectrum should exhibit two characteristic frequencies corresponding to the values of $\omega$ at which $\gamma_{c}$ and $\gamma_{d}$ enters into the visibility domains. This is confirmed in the next section in which we present our results.

\subsection{Results}

We have derived the stochastic gravitational wave spectrum $\hat{\Omega}_{\mathrm{sgw}}(\omega)$ along the lines described in the previous section. For a given value of $\omega$, the rate equation (3.14) is solved for $\hat{h}_{\star}(\omega)$. In order to keep track of the non-scaling loops appearing during the transition from the radiation era to the matter era, the loop distribution $t^{4} \mathcal{F}$ is numerically computed at each redshift $z$ by solving the Boltzmann equation of Ref. [52] [see Eq. (33) in this work]. The two-dimensional integrals appearing in both Eqs. (3.11) and (3.14) have been computed by means of the public CUBA library $[64,65]$ coupled to a Brent's method for solving the rate equation. Thermal history effects have been included in the cosmological variables $\chi(z), H(z)$ and $t(z)$ according to the Hindmarsh-Philipsen equation of state B $[57,66]$. The stochasticity threshold $\hat{h}_{\star}(\omega)$ is represented in the upper panel of figure 4 as a function of $f=\omega /(2 \pi)$ for a loop distribution having $G U=10^{-7}$. The three curves correspond to $\mathrm{GW}$ emission by one cusp, one kink, or one kink collision per loop oscillation. The corresponding spectra $\hat{\Omega}_{\mathrm{sgw}}(\omega)$ have been plotted in the lower panel of figure 4. As expected, the cusp case dominates over the kink by roughly an order of magnitude, which itself dominates kink collision by also an order of magnitude. All these spectra are similar in shape because they share very similar integration domains in the plane $(\gamma, z)$. Notice however the different slopes right to the peak. As we show below, they are a direct window on the microscopic nature of the GW emission event. The thin solid and short-dashed curves represent the respective contribution of GW events coming from loops in the matter era (solid) and from loops in the radiation era (short-dashed).

The behaviour of the stochastic strain threshold $\hat{h}_{\star}(\omega)$ can be understood by rewriting the left-hand side of the rate equation (3.14) as

$$
\mathcal{R}=\int_{0}^{z_{\star}} \mathrm{d} z\left[\frac{\chi(z)}{t(z)}\right]^{2} \frac{H_{0}}{H(z)} \frac{8 \pi}{(1+z)^{4}\left[H_{0} t(z)\right]^{2}} \int_{\max \left(\gamma_{1}, \gamma_{\star}\right)}^{\gamma_{\infty}} \mathrm{d} \gamma \frac{t^{4} \mathcal{F}(\gamma, z)}{\gamma} \Delta_{\text {beam }}(\omega, \gamma, z) .
$$

At high frequency, one expects $\gamma_{1}(\omega, z)<\gamma_{c}$ such that the rate $\mathcal{R}$ is dominated by small loops at small redshift (see figure 3 ). With such a crude approximation, replacing the beam with Eq. (3.15), one gets

$$
\mathcal{R} \propto(4 \pi)^{3-\frac{3}{\alpha}} \frac{t_{0}^{4} \mathcal{F}_{0}}{\hat{h}_{\star}^{3}}\left(\frac{H_{0}}{\omega}\right)^{6},
$$

where $\mathcal{O}(1)$ factors have been dropped and with $t_{0}^{4} \mathcal{F}_{0} \equiv t_{0}^{4} \mathcal{F}\left(\gamma<\gamma_{\mathrm{c}}, z=0\right)$. Solving $\mathcal{R}=$ $\omega /\left(2 \pi H_{0}\right)$ for $\hat{h}_{\star}$ gives

$$
\hat{h}_{\star}(\omega) \propto(4 \pi)^{1-\frac{1}{\alpha}}\left(t_{0}^{4} \mathcal{F}_{0}\right)^{1 / 3}\left(\frac{H_{0}}{\omega}\right)^{7 / 3}
$$

This approximate expression for $\hat{h}_{\star}$ has been represented as dotted lines in the upper panel of figure 4 and roughly reproduces the high frequency behaviour of $\hat{h}_{\star}(\omega)$ but overestimates the correct value at low frequencies.

For all sources, cusp, kink and collision, the spectrum $\Omega_{\mathrm{sgw}}(\omega)$ exhibits a knee at angular frequency $\omega_{\text {knee }}$ followed by a maximum at $\omega_{\text {peak }}$. As mentioned in section 3.2, these two features are the result of the scales $\gamma_{\mathrm{d}}$ and $\gamma_{\mathrm{c}}$ entering the loop visibility domains. From 

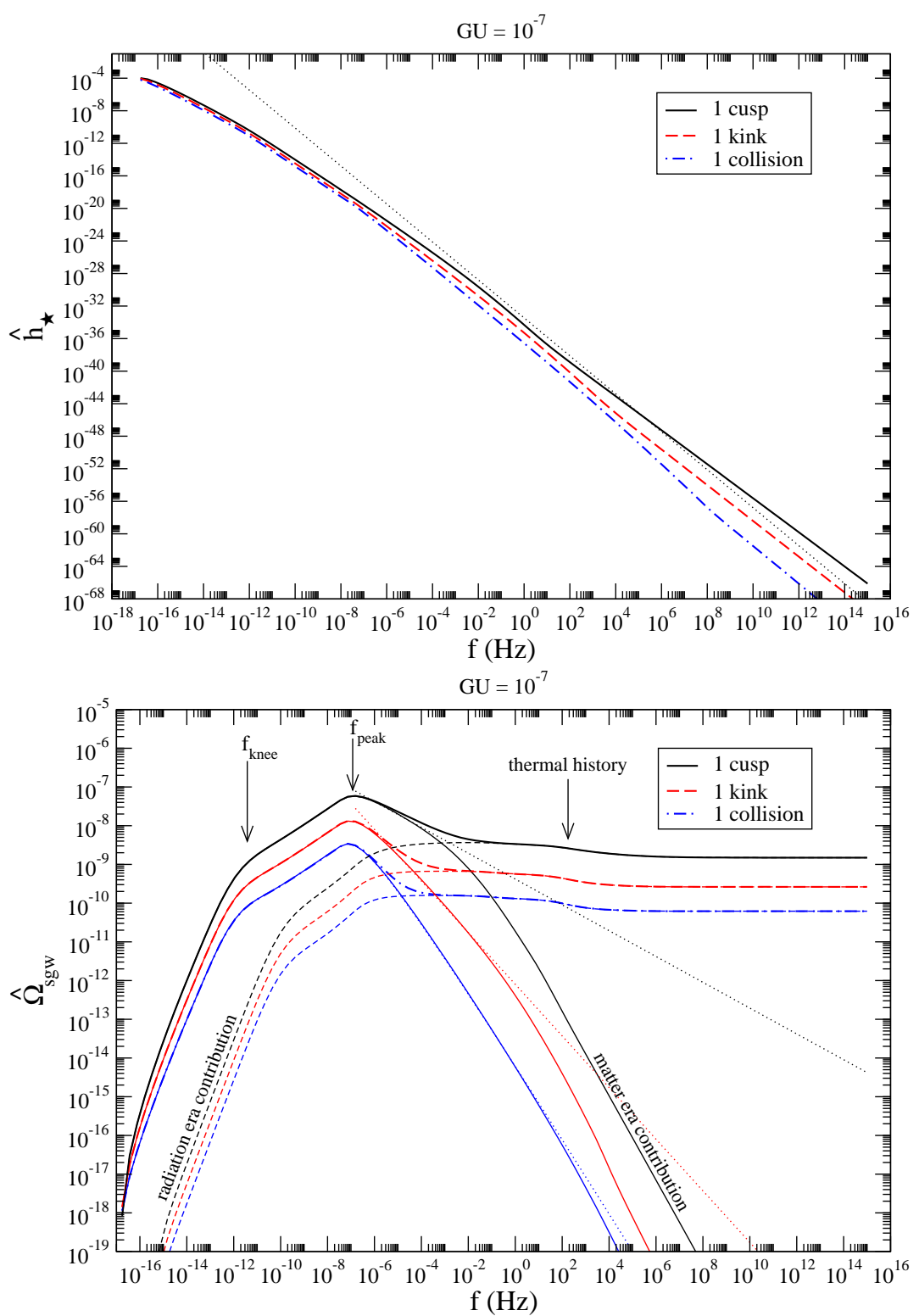

Figure 4. The upper panel represents the threshold strain $\hat{h}_{\star}(\omega)$, solution of Eq. (3.14), under which GW events are considered stochastic as a function of the observed frequency $f=\omega /(2 \pi)$. The thick solid line (black) is for one cusp, the thick dashed line (red) for one kink and the thick dotteddashed line (blue) for one kink collision, per loop oscillation. The lower panel shows the stochastic gravitational wave spectrum $\hat{\Omega}_{\mathrm{sgw}}(\omega)$ for the same three GW emission events. In addition, we have represented as thin solid and thin short-dashed lines the respective contribution of the matter era and radiation era loops to the overall spectrum. The peak is essentially produced by loops in the matter era while the high frequency plateau is induced by the loops in the radiation era. Dotted lines are analytic approximations (see text).

figure 3, a sufficient condition for $\gamma_{\mathrm{c}}$ to belong to the integration domain is $\gamma_{1}(\omega, z=0) \leq \gamma_{\mathrm{c}}$. 
Defining the angular frequency $\omega_{\mathrm{c}}$ at which these two quantities are equal, one gets

$$
\frac{\omega_{\mathrm{c}}}{H_{0}}=\frac{4 \pi}{t_{0} H_{0} \gamma_{\mathrm{c}}} \text {. }
$$

For $G U=10^{-7}$ and $\Upsilon=20$, one has $\omega_{\mathrm{c}} /(2 \pi) \simeq 2 \times 10^{-8} \mathrm{~Hz}$ which gives the correct order of magnitude for the peak frequency, see figure 4. However, a closer look to this figure reveals that the peak frequency depends on the type of GW burst, namely on the value of $\alpha$. In order to derive a better analytic approximation, let us consider $\hat{\Omega}_{\mathrm{sgw}}(\omega)$ around the maximum. From Eqs (3.11) and (3.19), it reads

$$
\hat{\Omega}_{\mathrm{sgw}}(\omega)=\frac{2(G U)^{2} \omega^{3}}{3 H_{0}^{3}} \int_{z_{\times}}^{z_{1}} \frac{\mathrm{d} z}{(1+z)^{2}} \frac{H_{0}}{H(z)} \int_{\gamma_{1}}^{\min \left(\gamma_{\star}, \gamma_{\infty}\right)} \frac{\mathrm{d} \gamma}{\gamma} t^{4} \mathcal{F}(\gamma, z) \hat{\mathcal{C}}^{2}(\omega, \gamma, z) \Delta_{\text {beam }}(\omega, \gamma, z) .
$$

As before, we can make the crude assumption that the integral over $\gamma$ is dominated by small loops at small redshift, and we keep only terms having $\gamma \leq \gamma_{\mathrm{c}}$ and $z<z_{\Lambda}$, where $z_{\Lambda}$ is an arbitrary cut-off. In order to keep the redshift terms of the integral simple, let us chose $z_{\Lambda}$ to be the redshift under which the cosmological constant dominates, i.e. $H\left(z<z_{\Lambda}\right) \simeq H_{0} \sqrt{\Omega_{\Lambda}}$. One gets

$$
\begin{aligned}
\hat{\Omega}_{\mathrm{sgw}}(\omega) & \propto(G U)^{2}\left(\frac{H_{0}}{\omega}\right)^{\frac{1}{\alpha}} z_{\Lambda} \frac{(4 \pi)^{1-\frac{1}{\alpha}}}{\left(t_{0} H_{0}\right)^{3+\frac{1}{\alpha}}} t_{0}^{4} \mathcal{F}_{0} \frac{\gamma_{\mathrm{c}}^{1-\frac{1}{\alpha}}}{1-\frac{1}{\alpha}}\left\{1-\left[\frac{\gamma_{1}(\omega, 0)}{\gamma_{\mathrm{c}}}\right]^{1-\frac{1}{\alpha}}\right\} \\
& \simeq(G U)^{2} \frac{t_{0}^{4} \mathcal{F}_{0}}{\left(t_{0} H_{0}\right)^{3+\frac{1}{\alpha}}} \frac{\left(4 \pi \gamma_{\mathrm{c}}\right)^{1-\frac{1}{\alpha}}}{1-\frac{1}{\alpha}}\left(\frac{H_{0}}{\omega_{\mathrm{c}}}\right)^{\frac{1}{\alpha}}\left[\left(\frac{\omega_{\mathrm{c}}}{\omega}\right)^{\frac{1}{\alpha}}-\frac{\omega_{\mathrm{c}}}{\omega}\right],
\end{aligned}
$$

in which we have dropped all $\mathcal{O}(1)$ factors and used the definition $(3.29)$ of $\omega_{\mathrm{c}}$. This formula is valid only for $\omega>\omega_{\mathrm{c}}$. In spite of the crude approximations made, the above expression is surprisingly accurate. The dependency in $\omega$ describes a maximum occurring at

$$
\frac{\omega_{\text {peak }}}{H_{0}} \simeq \frac{4 \pi \alpha^{\frac{\alpha}{\alpha-1}}}{t_{0} H_{0} \gamma_{\mathrm{c}}}=\frac{4 \pi \alpha^{\frac{\alpha}{\alpha-1}}}{t_{0} H_{0} \Upsilon}(G U)^{-(1+2 \chi)} .
$$

Notice that all the above expressions are regular for $\alpha \rightarrow 1$ and can be used for kink collisions as well: one has $\alpha^{\alpha /(\alpha-1)} \rightarrow e$ while the power laws in Eq. (3.31) becomes logarithmic functions. Equation (3.32) for the peak frequency can be compared to the exact numerical results of figure 4 . One finds $\omega_{\text {peak }} /(2 \pi)=1.3 \times 10^{-7} \mathrm{~Hz}$ which matches the exact numerical value. For kinks, $\omega_{\text {peak }} /(2 \pi)=8.3 \times 10^{-8} \mathrm{~Hz}$ for an exact value at $8.7 \times 10^{-8}$ and for collisions one gets $\omega_{\text {peak }} /(2 \pi)=6.7 \times 10^{-8} \mathrm{~Hz}$ instead of $7.5 \times 10^{-8} \mathrm{~Hz}$. The $\omega$-dependency of Eq. (3.31) around the maximum is such that

$$
\left.\hat{\Omega}_{\mathrm{sgw}}\left(\omega \gtrsim \omega_{\text {peak }}\right)\right|_{\alpha \neq 1} \propto \omega^{-\frac{1}{\alpha}},\left.\quad \hat{\Omega}_{\mathrm{sgw}}\left(\omega \gtrsim \omega_{\text {peak }}\right)\right|_{\alpha=1} \propto \omega^{-1} \ln \omega .
$$

These analytic approximations have been represented in the lower panel of figure 4 as dotted curves for $\omega>\omega_{\mathrm{c}}$ and appear to fit relatively well the decrease of $\hat{\Omega}_{\mathrm{sgw}}(\omega)$ just after its maximum. As a result, there is a small window in frequencies, for $\omega \gtrsim \omega_{\text {peak }}$, in which the slope of the stochastic gravitational wave spectrum directly reflects the type of GW bursts occurring along the cosmic string loops. Plugging Eq. (3.32) into Eq. (3.31) allows us to derive the dependency of the maximal power with respect to the string tension. One gets

$$
\hat{\Omega}_{\mathrm{sgw}}\left(\omega_{\text {peak }}\right) \propto(G U)^{2} t_{0}^{4} \mathcal{F}_{0} \gamma_{\mathrm{c}} \propto(G U)^{2} \frac{\gamma_{\mathrm{c}}^{2 \chi-1}}{\gamma_{\mathrm{d}}} \propto(G U)^{4 \chi^{2}},
$$


where we have used the approximate relation $t_{0}^{4} \mathcal{F}_{0} \propto \gamma_{\mathrm{c}}^{2 \chi-2} / \gamma_{\mathrm{d}}$ derived in Ref. [52]. Since, for not too small values of $G U$, the peak is dominated by matter era loops, the value $\chi=\chi_{\mathrm{M}}$ should be used in this expression.

Concerning the knee in the spectrum, it corresponds to the change of behaviour of the loop distribution at $\gamma=\gamma_{\mathrm{d}}$ (see figure 1). As a result, all of the previous approximations can equally be applied to determine the knee frequency by formally replacing $\gamma_{\mathrm{c}}$ by $\gamma_{\mathrm{d}}$. One obtains

$$
\omega_{\mathrm{knee}} \simeq \frac{4 \pi \alpha^{\frac{\alpha}{\alpha-1}}}{t_{0} H_{0} \gamma_{\mathrm{d}}}=\frac{4 \pi \alpha^{\frac{\alpha}{\alpha-1}}}{t_{0} H_{0} \Gamma}(G U)^{-1}
$$

with

$$
\hat{\Omega}_{\mathrm{sgw}}\left(\omega_{\text {knee }}\right) \propto(G U)^{2} t_{0}^{4} \mathcal{F}\left(\gamma_{\mathrm{d}}, 0\right) \gamma_{\mathrm{d}} \propto(G U)^{2} \gamma_{\mathrm{d}}^{2 \chi-2} \propto(G U)^{2 \chi} .
$$

These approximations cease however to be valid as soon as the radiation-era loops contribute significantly to the peak.

The radiation-era loops are expected to be the main source of GW in the high frequency limit. Figure 3 indeed shows that, at high frequency, the domains in which the loop distribution is maximal, namely for $\gamma \leq \gamma_{\mathrm{c}}$, end up being in the radiation-era. This can be explicitly seen by plugging Eq. (3.28) into the definition of $z_{\times}$in Eq. (3.23):

$$
z_{\times} \propto\left(t_{0}^{4} \mathcal{F}_{0}\right)^{-\frac{1}{3}}\left(\frac{\omega}{H_{0}}\right)^{\frac{1}{3 \alpha}}
$$

For $\omega / H_{0}$ large enough, $z_{\times}$lies in the radiation-era. Keeping only loops having $\gamma \leq \gamma_{\mathrm{c}}$ and taking all the redshift dependent terms in Eq. (3.30) to be in the radiation era, one recovers that the high frequency limit is frequency independent [63]

$$
\hat{\Omega}_{\mathrm{sgw}}(\omega) \propto \Omega_{\mathrm{rad}} \mathcal{Q}\left(z_{\mathrm{c}}\right) \frac{\gamma_{\mathrm{c}}^{2 \chi-1}}{\gamma_{\mathrm{d}}} \propto \Omega_{\mathrm{rad}} \mathcal{Q}\left(z_{\mathrm{c}}\right)(G U)^{4 \chi^{2}}
$$

where $z_{\mathrm{c}}$ is a redshift at which $\gamma_{1}\left(z_{\mathrm{c}}\right)=\gamma_{\mathrm{c}}$ is satisfied. The quantity $\mathcal{Q}(z) \equiv q_{0}^{4 / 3} g(z) /\left[q(z)^{4 / 3} g_{0}\right]$ denotes the change in energetic $(g)$ and entropic $(q)$ relativistic degrees of freedom between $z$ and today. The presence of $\mathcal{Q}\left(z_{\mathrm{c}}\right)$ justifies the smooth steps visible in figure 4 and labelled as "thermal history". Depending on the redshift $z$ at which the GW event has been emitted, the amplitude of the received signal is modulated by $\mathcal{Q}(z)$, through its effect on $H(z), \chi(z)$ and $t(z)$. As a result, and as it is the case for GW produced during inflation, cosmic string loops could be used to perform GW tomography of the radiation-era [67,68]. Although the amplitude of the high frequency plateau scales as $(G U)^{4} \chi^{2}$, which is the same function as the peak amplitude in Eq. (3.34), one has to use the radiation era value $\chi=\chi_{\mathrm{R}}$ in Eq. (3.38). Therefore, the peak and the plateau do not scale in the exact same way with respect to $G U$.

In figure 5 , we have plotted various spectra $\hat{\Omega}_{\mathrm{sgw}}(\omega)$, still for one cusp, one kink and one collision event per loop oscillation, for different values of the string tension $G U$. For the cusp case (upper right panel) the dotted lines are the analytic approximations defined in Eqs. (3.32) and (3.34). As can be seen on this plot, the approximations are accurate down to $G U \simeq 10^{-11}$ while for lower string tension we find that Eq. (3.34) underestimates the peak amplitude. Let us stress than Eq. (3.32) for the peak frequency remains accurate. In the lower right panel of figure 5 , we have plotted the numerical value of $\hat{\Omega}_{\mathrm{sgw}}\left(\omega_{\text {peak }}\right)$ as a function of $G U$. In this plot, the dotted lines are the approximations of Eq. (3.34) using either $\chi=\chi_{\mathrm{M}}$ (steeper slope) or $\chi=\chi_{\mathrm{R}}$. Very low string tensions are better fitted with the 

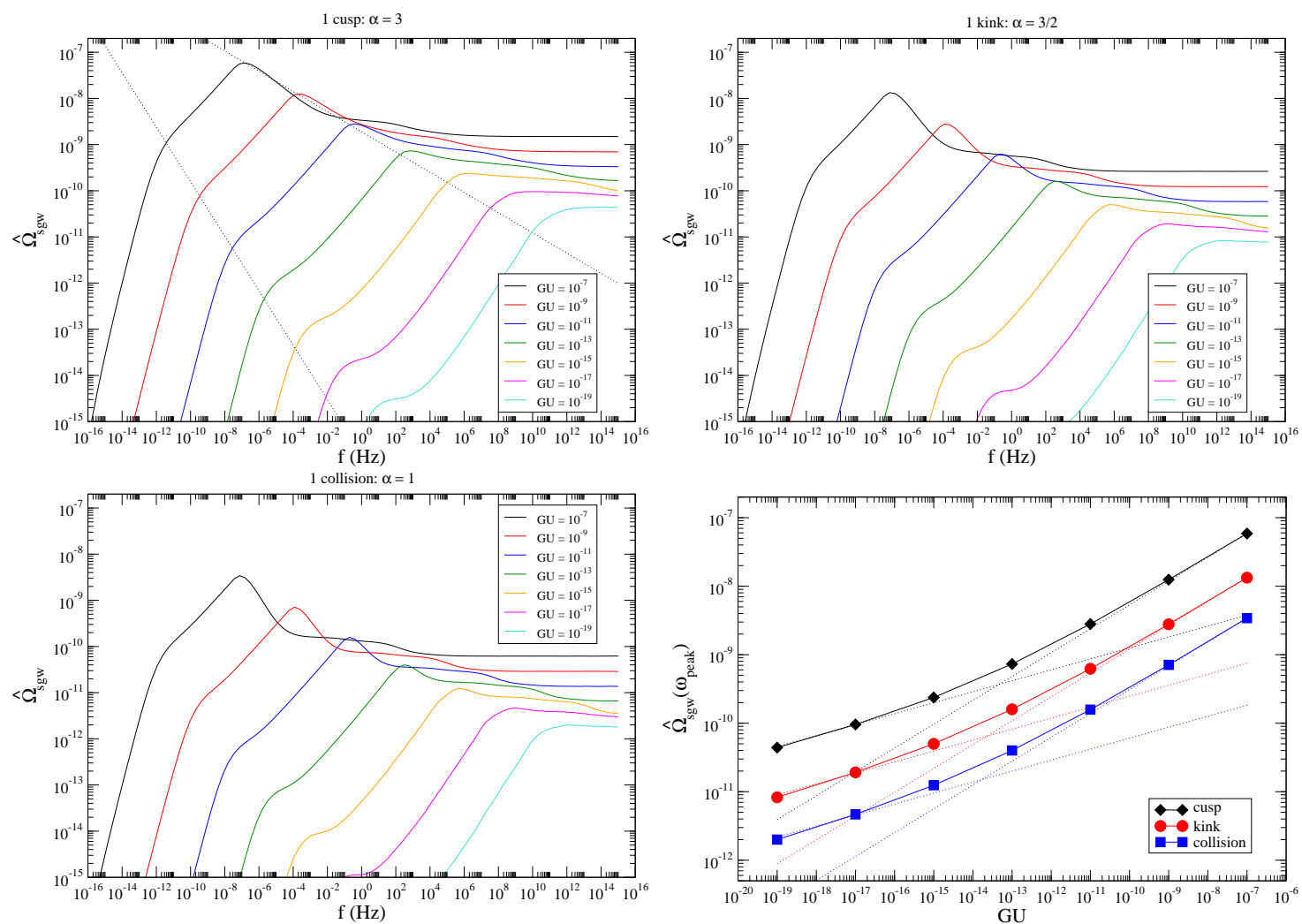

Figure 5. Stochastic GW spectra for one cusp (upper left), one kink (upper right) and one collision (lower left) event per loop oscillation as a function of the string tension $G U$. The dotted lines in the upper left panel are the analytic approximations of Eqs. (3.32) and (3.34). The lower right panel shows the maximal amplitude $\hat{\Omega}_{\mathrm{sgw}}\left(\omega_{\text {peak }}\right)$ as a function of $G U$ together with the analytic approximations as dotted lines (see text).

radiation era value for $\chi$ suggesting that radiation-era loops significantly contribute to the overall peak. The shape of the spectrum for these values of $G U$ is also severely distorted: the maximum occurs at very high frequency and it progressively becomes undistinguishable from the plateau. Notice however the different behaviour of the knee frequency and amplitude.

\subsection{Comparison with previous works}

Because we have paid special attention to consider a realistic loop distribution, it is difficult to compare our results to those having used single-sized distributions or production functions [28-36]. However, our results could be compared to those of Refs. [37, 63, 69] which have considered non-trivial loop distributions. The overall shape of our spectrum matches with the one presented in these works, namely all of them exhibit a plateau at high frequencies, a maximum and a fast decay at low frequencies. Concerning the relative amplitude of cusp versus kink, our spectra have a more pronounced hierarchy that the ones computed in Ref. [63] but this could be the result of some specific numerical values chosen for their coefficient $c_{\alpha}$ in Eq. (3.18). The presence of a knee in the spectrum is somehow reminiscent with the two-scales loop model of Ref. [69], although our spectra are significantly different in shapes and amplitude in this case. Because Ref. [69] uses more than five parameters to 


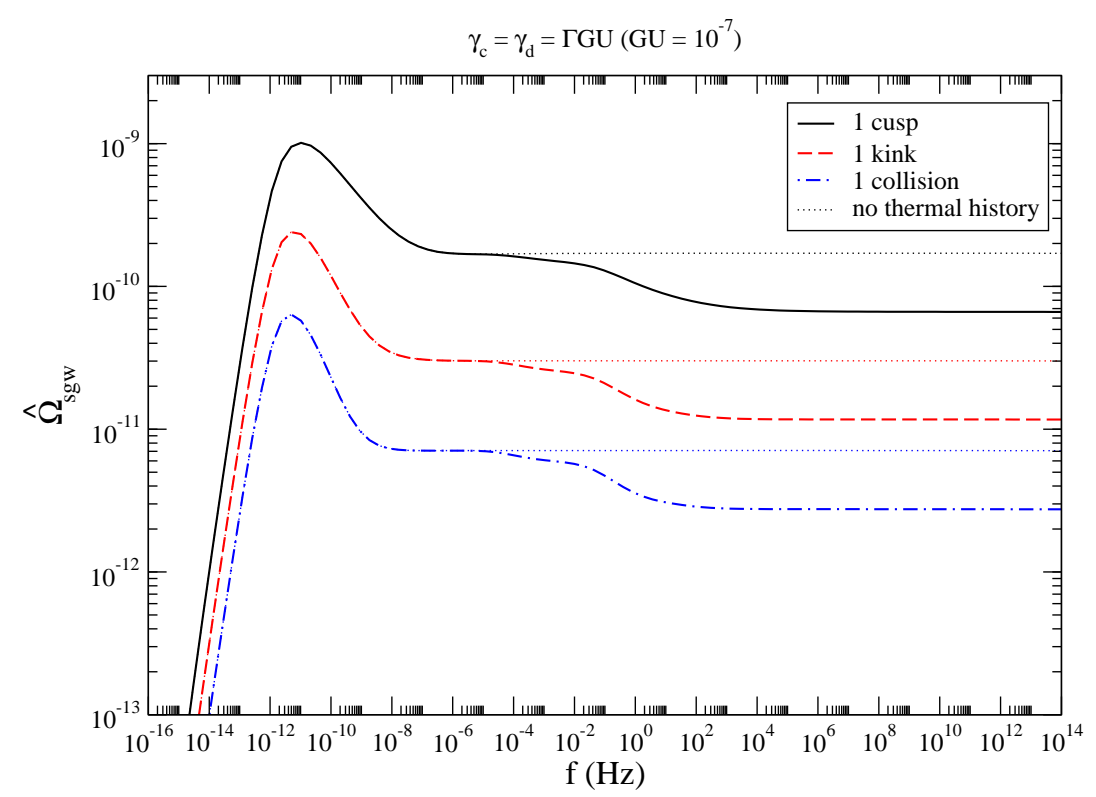

Figure 6. Stochastic GW spectra for one cusp, one kink and one collision event per loop oscillation for a scaling loop distribution having $\gamma_{\mathrm{c}}=\gamma_{\mathrm{d}}=\Gamma G U$ with $\Gamma=50$ and $G U=10^{-7}$. Notice that the physical spectra are $\Omega_{\mathrm{sgw}}(\omega)=c_{\alpha}^{2} \hat{\Omega}_{\mathrm{sgw}}(\omega)$ where $c_{\alpha}$ is given in Eq. (3.18). The dotted curves are derived without thermal history by assuming a pure radiation era without any changes in the number of relativistic degrees of freedom.

model the spectrum, it is possible that some combination of them, and a multi-scale loop model, could reproduce our result but we have not attempted further comparison.

More interestingly the results of Ref. [37] are based on a realistic loop distribution inferred from Nambu-Goto simulations. Their spectrum has been derived without thermal history effects and for cusp events only. Up to the thermal effects in the high frequency part, the main differences are that the peak of their spectrum matches the knee in ours. As discussed earlier, the overall maximum of $\hat{\Omega}_{\mathrm{sgw}}(\omega)$ is associated with the gravitational backreaction scale $\gamma_{\mathrm{c}}$ in the loop distribution whereas the knee traces the gravitational emission length scale $\gamma_{\mathrm{d}}$. The results of Ref. [37] have been derived under the assumption $\gamma_{\mathrm{c}}=\gamma_{\mathrm{d}}=\Gamma G U$. In figure 6 , we have therefore plotted the cusp, kink and collision dimensionless spectra $\hat{\Omega}_{\mathrm{sgw}}(\omega)$ in the particular case $\gamma_{\mathrm{c}}=\gamma_{\mathrm{d}}$ and without thermal history effects. As expected, the peak and knee previously visible in figure 4 are merged and the cusp spectrum can be directly compared to the one derived in Ref. [37] (see figure 13) provided we estimate $c_{3}$. From Eq. (3.18), taking $\beta=1$ and $\eta_{\mathrm{s}}$ from Eq. (2.14), one gets $c_{3} \simeq 7$ such that one has to multiply the cusp spectrum of figure 6 by $c_{3}^{2}$ to get the physical one. For $G U=10^{-7}$, we get the peak frequency at $\omega_{\text {peak }} /(2 \pi) \simeq 1.0 \times 10^{-11} \mathrm{~Hz}$, which, up to a factor of two, matches the one of figure 13 in Ref. [37]. For the maximum amplitude, we get $\Omega_{\mathrm{sgw}}\left(\omega_{\text {peak }}\right) \simeq 5 \times 10^{-8}$, to be compared to a value around $6 \times 10^{-8}$ as it can be inferred from their figure 13. Both spectra are therefore within a ten percent agreement for the amplitude when derived under the condition $\gamma_{\mathrm{d}}=\gamma_{\mathrm{c}}$. Let us notice however that the power law exponents of the loop distribution between Ref. [25] and Ref. [37] are slightly different. In terms of our parameter $\chi$, for the radiation era, Ref. [37] uses a value of $\chi_{\mathrm{R}}=0.25$ (and $\chi_{\mathrm{M}}=0.5$ ) such that this agreement is degraded for the lower values of $G U$. For instance, for $G U=10^{-11}$, we find an agreement 
only up to a factor of 6 for the amplitude. For much lower values of $G U$, as can be checked in our figure 5, relaxation effects, which are encoded in our Boltzmann approach, becomes significant and the loop distributions cannot be straighforwardly compared anymore.

Although this agreement can be considered as a validation of our numerical method, let us stress the importance of considering the two gravitational length scales $\gamma_{\mathrm{d}}$ and $\gamma_{\mathrm{c}}$ separated. As can be seen in figure 5, the overall peak of our spectrum is significantly higher than the knee while its amplitude decreases with $G U$ much slower than the amplitude at the knee frequency. This is expected from the shape of the loop distribution in figure 1 . The size and number of loops of size $\gamma_{\mathrm{c}}$ is significantly boosted compared to those of size $\gamma_{\mathrm{d}}$ when $G U \rightarrow 0$. Let us also stress the importance of thermal history effects which lower the plateau by a factor $\mathcal{O}(3)$, especially around the LIGO frequencies.

Finally, up to our knowledge, the collision spectrum has not been derived before. Its importance will be discussed in the next section in which we explore the range of remaining theoretical uncertainties on the loop microstructure.

\section{Loop microstructure and constraints}

The string microstructure affects the observable GW spectrum through the coefficients $c_{\alpha}$ in Eq. (3.18). The precise determination of these coefficients is out of the scope of the current work and would require a precise examination of various correlation functions along the string worldsheet. However, we expect the order of magnitude for $c_{\alpha}$ to be relatively accurate and in the following we will be using the fiducial values

$$
\beta=1, \quad v_{ \pm}^{2}=0.5
$$

and $\eta_{\mathrm{s}}$ given by Eq. (2.14). As discussed in the introduction, the number of cusps, kinks and collisions per loop oscillations is currently unknown and constitutes the main source of theoretical uncertainties on the observable spectrum. Denoting by $N_{\mathrm{c}}, N_{\mathrm{k}}$ and $N_{\mathrm{kk}}$ the number of cusps, kinks and collisions appearing per loop oscillation, the final spectrum $\hat{\Omega}_{\mathrm{sgw}}(\omega)$ can be obtained by the weighted contributions of each of the spectra derived in the previous section. Notice however that the stochasticity threshold $\hat{h}_{\star}$ has also to be recomputed for each value of $N_{\mathrm{c}}, N_{\mathrm{k}}$ and $N_{\mathrm{kk}}$ and that these numbers may depend on the loop size $\gamma$.

\subsection{Gravitational wave emission bound}

The numbers $N_{\mathrm{c}}, N_{\mathrm{k}}$ and $N_{\mathrm{kk}}$ cannot take arbitrarily large values as the total power emitted by each loop under the form of GW is given by $P_{\mathrm{gw}}=\Gamma G U^{2}$. For one $\mathrm{GW}$ emission event per loop oscillation, in the local wave zone around each loop, one has $P_{\mathrm{gw}}=\rho_{\mathrm{gw}}^{(\mathrm{o})} 4 \pi r^{2} \Delta_{\text {beam }}$. From Eq. (2.19), one gets

$$
P_{\mathrm{gw}}=\frac{G U^{2}}{\ell^{2}} \sum_{n=1}^{+\infty} \varpi_{n}^{2} \overline{\mathcal{C}}^{2}\left(\varpi_{n}, \ell\right) \Delta_{\text {beam }}\left(\varpi_{n}, \ell\right)
$$

This expression is valid for any sources, but one expects the cusp, kink or collision waveforms to dominate over all the other terms in the high frequency regime, i.e., for $n \gg 1$, see section 2.2. On the contrary, for the first values of $n$, one expects $\overline{\mathcal{C}}^{2}$ to be dominated by the loop oscillation modes. Therefore, we define a threshold, $n_{\text {cut }}$, above which GW emission is dominated by cusp, kink or collision events only. A precise value for $n_{\text {cut }}$ is not known, 
although a possible number inferred from the simulations of Ref. [39] could be taken as $\mathcal{O}(10)$. In the following, we will be using the safe value $n_{\text {cut }}=5$, which might underestimate the maximal allowed numbers of cusp, kinks and collision events per loop oscillation. As a result, the GW power emitted by the string microstructure only (cusp, kinks or collisions) per loop oscillation reads

$$
P_{\mathrm{gw}}^{(\alpha)}=N_{(\alpha)} \frac{G U^{2}}{\ell^{2}} \sum_{n=n_{\mathrm{cut}}}^{+\infty} \overline{\mathcal{C}}_{(\alpha)}^{2}\left(\varpi_{n}, \ell\right) \Delta_{\text {beam }}^{(\alpha)}\left(\varpi_{n}, \ell\right)
$$

where $N_{(\alpha)}, \overline{\mathcal{C}}_{(\alpha)}^{2}$ and $\Delta_{\text {beam }}^{(\alpha)}$ are for the cusp, kink and collision waveforms $\left(N_{(3)} \equiv N_{\mathrm{c}}\right.$, $\left.N_{(3 / 2)} \equiv N_{\mathrm{k}}, N_{(1)} \equiv N_{\mathrm{kk}}\right)$. Because $P_{\mathrm{gw}}^{(\alpha)} \leq P_{\mathrm{gw}}$, using Eqs. (3.15) and (3.16), one obtains

$$
N_{(\alpha)} \leq\left(\frac{\sqrt{3}}{2}\right)^{1-\frac{1}{\alpha}} \frac{(4 \pi)^{\frac{2}{\alpha}} \Gamma}{b_{\alpha} c_{\alpha}^{2} \sum_{n=n_{\text {cut }}}^{+\infty} \frac{1}{n^{1+\frac{1}{\alpha}}}}
$$

Using the fiducial values mentioned before, with $\Gamma=50$, one gets

$$
N_{\mathrm{c}} \leq 11, \quad N_{\mathrm{k}} \leq 257, \quad N_{\mathrm{kk}} \leq 4459 .
$$

As can be seen in figure 4, a large number of kinks or collision events per loop oscillation may therefore allow the total stochastic GW background to be dominated by kinks or collisions instead of the naively expected cusps. For this reason, in the following, we devise four prototypical scenarios according to the hierarchy between the number of cusps and kinks present on the loops.

\subsection{Prototypical models of string microstructure}

In the following, we will always assume that kinks are created in pairs, as it is observed in Nambu-Goto simulations such that $N_{\mathrm{kk}}=N_{\mathrm{k}}^{2} / 4$. Under this assumption, the number of kinks per loop oscillation is actually bounded by the maximal numbers of collisions and one gets $N_{\mathrm{k}} \leq 133$.

The first prototypical model assumes $N_{\mathrm{c}}=2$ and $N_{\mathrm{k}}=0$. It is motivated by the results of Ref. [45]. If GW backreaction is a very efficient process, loops microstructure is erased and the smooth loops tend to develop two cusps per oscillations. If the larger loops do not have too many kinks, $N_{\mathrm{k}} \ll \mathcal{O}(10)$, the overall stochastic GW background is dominated by cusps only. One could consider this case as the standard lore. We will refer to this model as $2 \mathrm{C}$ (2 cusps).

The second scenario assumes $N_{\mathrm{c}}=0$ and a not too large number of kinks: $N_{\mathrm{k}}^{2} / 4 \ll$ $\mathcal{O}\left(10^{-1}\right) N_{\mathrm{k}}$. This condition ensures that the stochastic GW background is always dominated by kinks and not by collisions. We choose $N_{\mathrm{k}}<20$. Such a scenario may be motivated by the results of Refs. [46, 47] and would describe the cases for which GW backreaction does not smooth the string microstructure. We will refer to this model as "LNK" (low number of kinks). The third model is the same as the previous one but assumes $20 \leq N_{\mathrm{k}} \leq 133$ such that the stochastic GW spectrum is dominated by collision events. This model will be refereed to as "HNK" (high number of kinks).

Finally, we also discuss a model in which loops smaller than the gravitational backreaction length scale $\gamma \leq \gamma_{\mathrm{c}}$ are smooth and develop two cusps per oscillation whereas the larger 


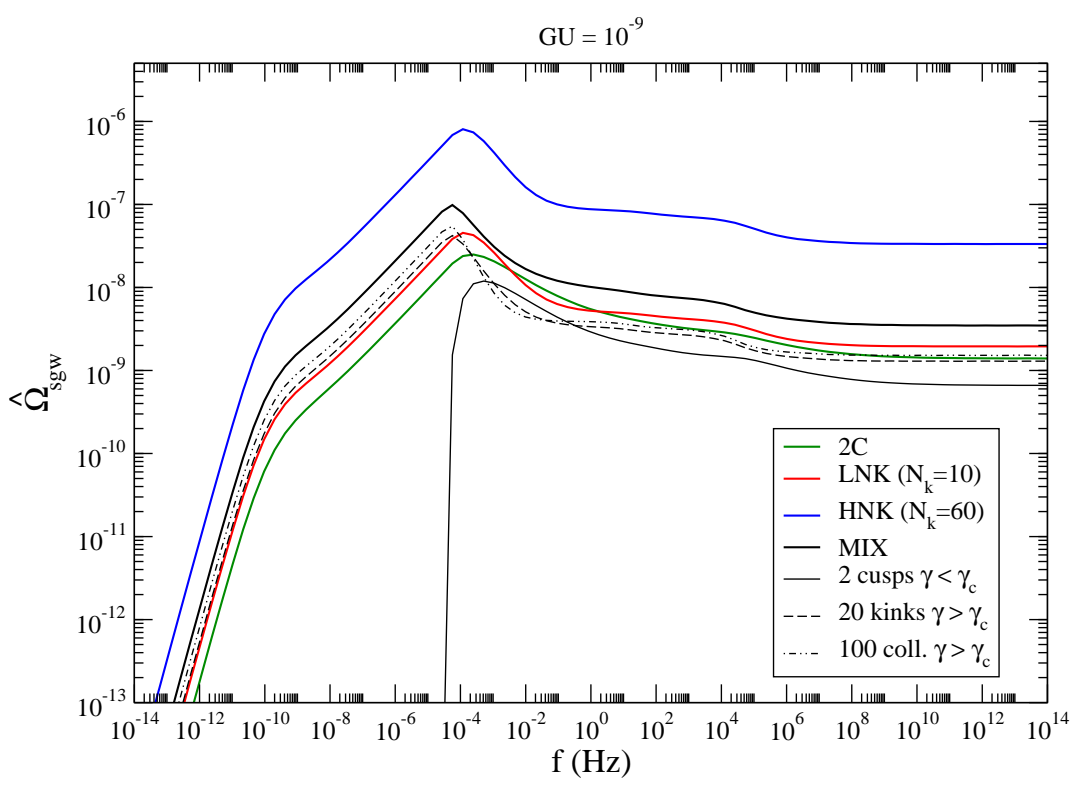

Figure 7. Stochastic GW spectra for the prototypical models having two cusps (2C), a low number of kinks $N_{\mathrm{k}}=10$ (LNK), and a high number of kinks $N_{\mathrm{k}}=60(\mathrm{HNK})$ per loop oscillation. The scenario labelled as MIX has two cusps for loops smaller than the gravitational backreaction length $\gamma \leq \gamma_{\mathrm{c}}$ and only kinks for larger loops (here $N_{\mathrm{k}}=20$ ).

ones $\gamma>\gamma_{\mathrm{c}}$ have only kinks. For those, we simply assume that $N_{\mathrm{k}} \leq 133$ and this scenario may be viewed as a scale-dependent mixture of $2 \mathrm{C}$, LNK and HNK. It will be referred to as "MIX" in the following.

In figure 7 , we have plotted the typical stochastic GW spectra $\hat{\Omega}_{\mathrm{sgw}}$ for these four models. Their shape can be understood from the spectra of individual events derived in section 3.3. For the MIX scenario, we have separated the contribution coming from cusps, kinks and collisions. Because the low frequency part of the spectrum is generated by large loops, the cusp contribution rapidly vanishes for $\omega<\omega_{\text {peak }}$.

Because $N_{\mathrm{k}}$ can vary over two orders of magnitude between these models, it is not possible to extract an unique model independent constraint on $G U$ from the current stochastic GW limits. For this reason, in the next section, we perform a Bayesian analysis of the parameter space $\left(G U, N_{\mathrm{k}}\right)$ for each of these scenarios. Marginalising over $N_{\mathrm{k}}$ within the appropriate prior range allows us to obtain a robust constraint on $G U$.

\subsection{Observational constraints}

The prototypical models of string microstructure presented in the previous section have been compared to the existing bounds on the stochastic gravitational wave background. For this purpose, we have used the recent results of the LIGO collaboration at $f=25 \mathrm{~Hz}$ presented in Ref. [70], as well as the ones coming from the European Pulsar Timing Array (EPTA) at $f=31.7 \mathrm{nHz}$ in Ref. [71].

\subsubsection{LIGO and EPTA data}

Unfortunately, up to our knowledge, none of these works have provided a public likelihood based on the frequencies that have been sampled to search for stochastic GW. As a result, 

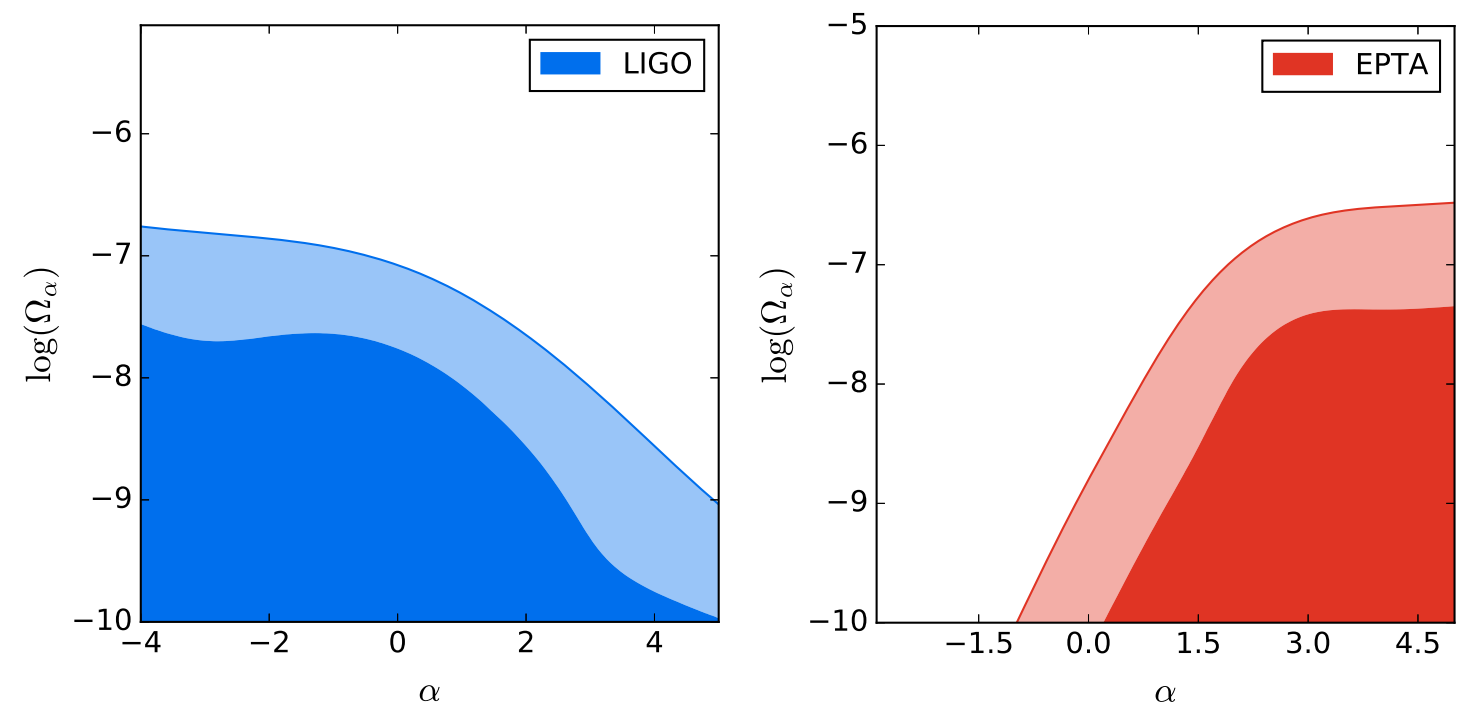

Figure 8. One- and two-sigma contour of the two-dimensional posterior distribution obtained by nested sampling of our toy LIGO (left) and EPTA (right) likelihoods. The resulting confidence intervals are found to reproduce well the results of Refs. [70, 71].

we have resorted to a basic two-dimensional Gaussian likelihood fitting of their two-sigma upper limit in the plane $\left(\Omega_{\alpha}, \alpha\right)$ where

$$
\Omega_{\mathrm{sgw}}(f) \equiv \Omega_{\alpha}\left(\frac{f}{f_{0}}\right)^{\alpha},
$$

$f_{0}$ being the reference frequency of the detector. In order to test our toy likelihood, we have sampled the parameter space $\left(\Omega_{\alpha}, \alpha\right)$ using the nested sampling algorithm MULTINEST [72-75] and extracted the one- and two-sigma contours of the associated two-dimensional posterior distributions. They have been plotted in figure 8 for both the LIGO and EPTA data. Our likelihood reproduces well the confidence interval plotted in figure 2 of Ref. [70] and in figure 14 of Ref. [71].

Let us stress the importance of keeping the spectral index $\alpha$ in the inference problem. As can be seen in figure 8 , the two-sigma upper limit on the amplitude $\Omega_{\alpha}$ may change by more than two orders of magnitude according to $\alpha$. This is particularly relevant for cosmic strings. As one can check in figure 7, the slope of the spectrum at the LIGO and EPTA frequencies, $f_{0}=25 \mathrm{~Hz}$ and $f_{0}=31.7 \mathrm{nHz}$, respectively, depends on all the string parameters, and in particular $G U$.

\subsubsection{Constraints on the string tension}

For the four prototypical models discussed earlier, namely 2C, LNK, HNK and MIX, we can evaluate both $\Omega_{\alpha}$ and $\alpha$ at the two frequencies $f_{0}=25 \mathrm{~Hz}$ and $f_{0}=31.7 \mathrm{nHz}$, corresponding to LIGO and EPTA, respectively. Plugging these numbers into our toy likelihood allows us, in return, to test the ability of each model to satisfy the LIGO and EPTA bounds.

In practice, we have sampled the parameter space associated with each model by using the nested sampling algorithm MULTINEST. For instance, for the model $2 \mathrm{C}$, the only parameter 

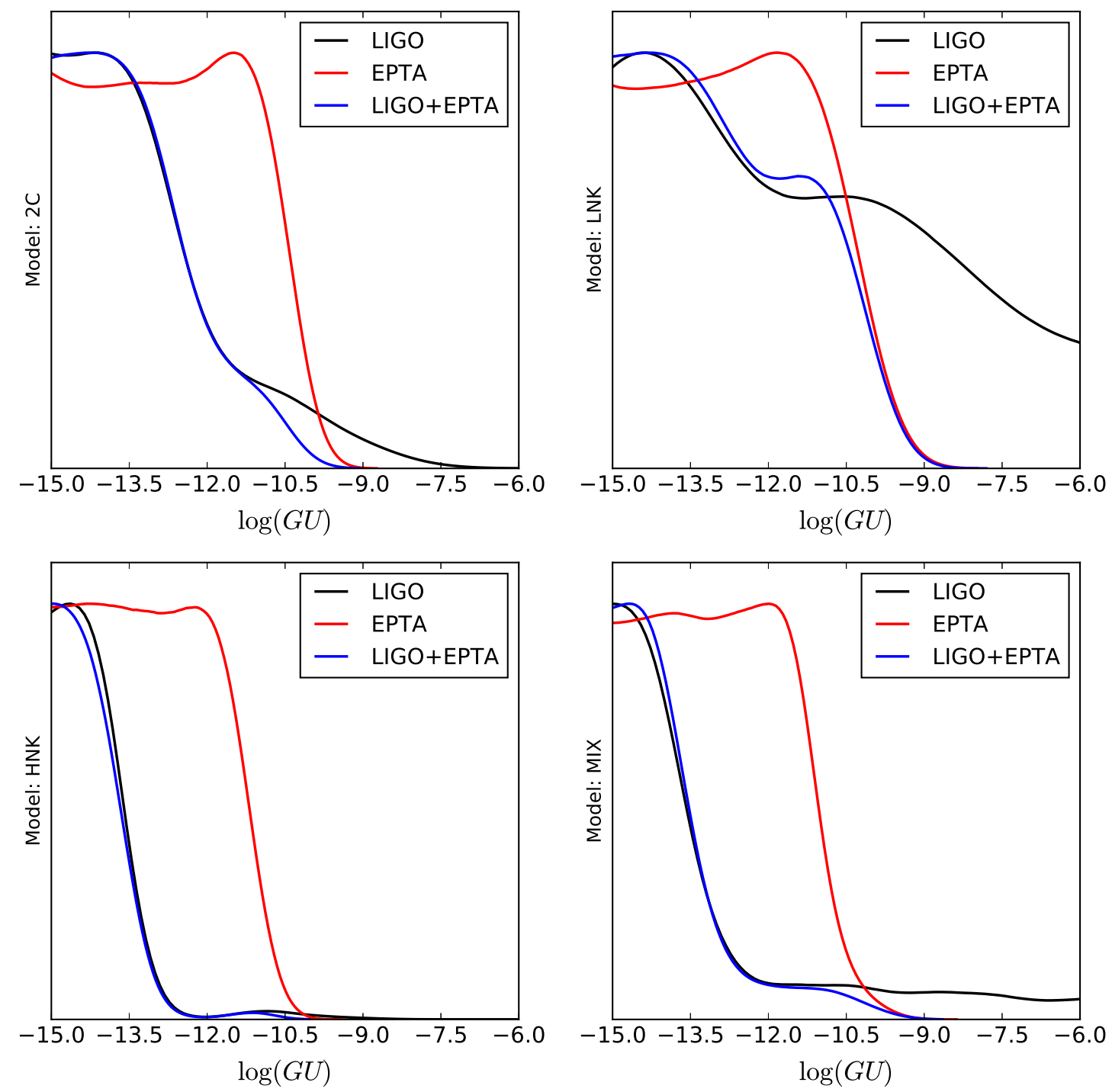

Figure 9. One dimensional marginalized posterior probability distribution for the string tension $G U$ associated with the microstructure models 2C, LNK, HNK and MIX. Apart for LNK, and to some extend MIX, all the models are strongly constrained by both LIGO and EPTA.

is $G U$, for which we have chosen a Jeffreys' prior as $\log (G U) \in[-15,-6]$. For the other models, LNK, HNK and MIX, we have kept the same prior for $G U$ and considered a flat prior for the number of kinks $N_{\mathrm{k}}$ in the ranges discussed in section 4.2. Although the dimensionality of the problem is small, two at maximum, calculating all the cusps, kinks and collisions part of the spectra amounts for about ten seconds of computation rendering the whole analysis numerically heavy. These difficulties have been overcome by using the MPI-parallelized version of MULTINEST over a 5000 live points and we have stopped the sampling for an expected error on the Bayesian evidence at $10^{-4}$. This typically corresponds to a total number of samples around $10^{4}$, which is accurate enough to determine the marginalized posterior in a two-dimensional parameter space [76]. 

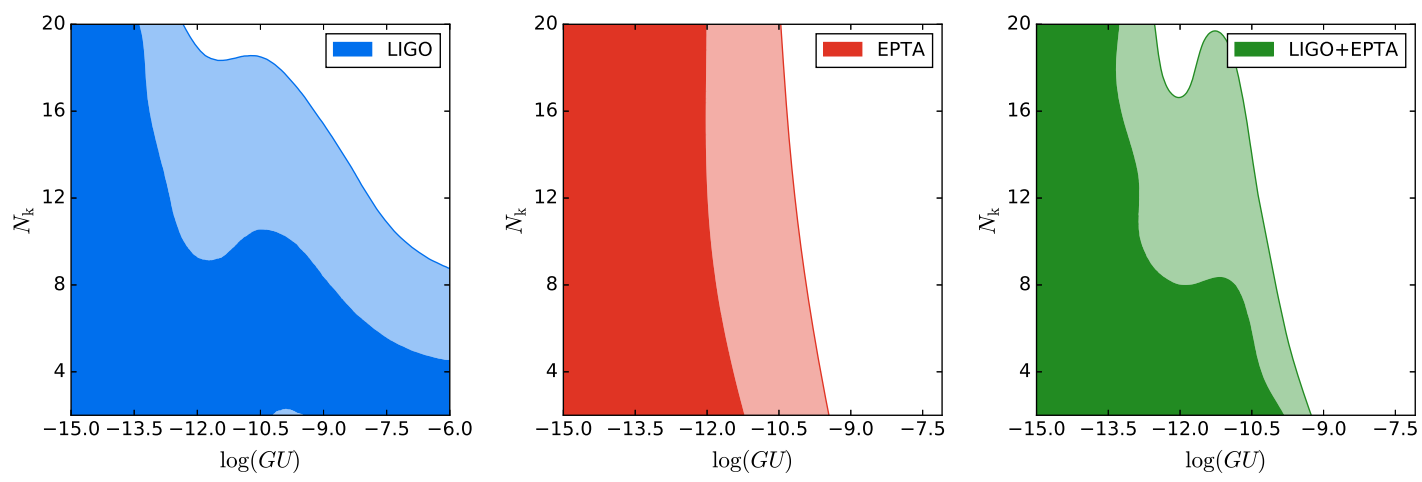

Figure 10. One- and two-sigma contours of the posterior probability distributions in the plane $\left[N_{\mathrm{k}}, \log (G U)\right]$ for the low number of kinks microstructure model LNK. For $N_{\mathrm{k}}<8$, there is no constraints coming from the LIGO bounds.
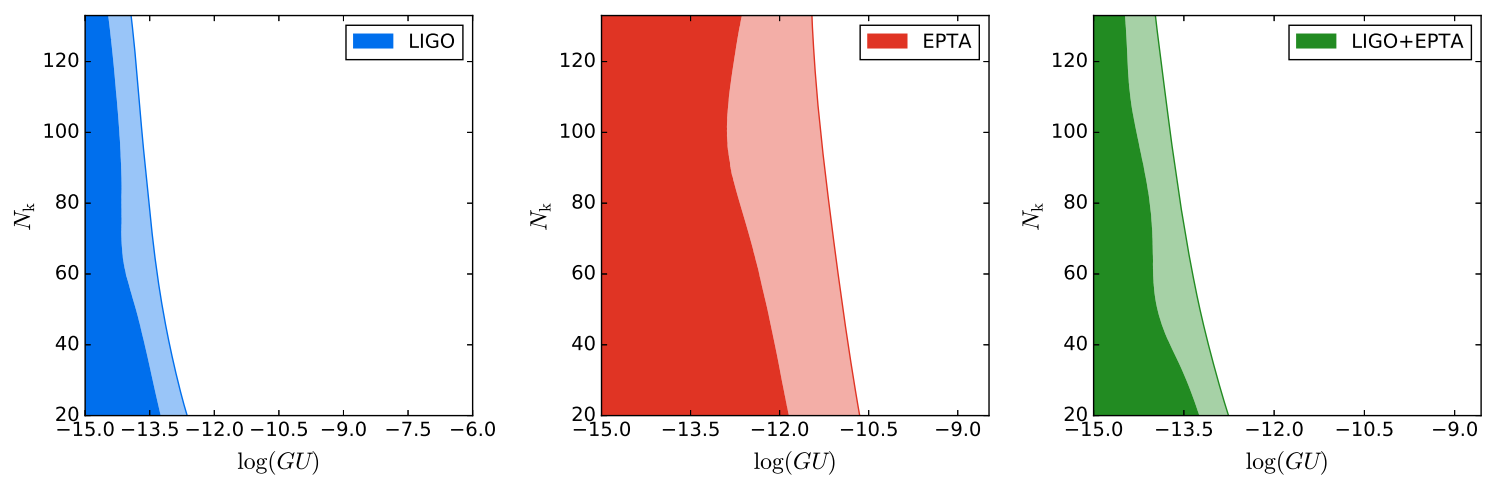

Figure 11. One- and two-sigma contours of the posterior probability distributions in the plane $\left[N_{\mathrm{k}}, \log (G U)\right]$ for the high number of kinks microstructure model HNK. This scenario is severely constrained by LIGO.

The one-dimensional posteriors for $G U$ have been represented in figure 9. Notice that, for the models LNK, HNK and MIX, these posteriors are obtained by marginalization over $N_{\mathrm{k}}$. The corresponding one- and two-sigma contours of the two-dimensional posteriors in the plane $\left[\log (G U), N_{\mathrm{k}}\right]$ have been plotted in figures 10, 11 and 12. Among other effects discussed below, the bounds on $G U$ become stronger for higher values of $N_{\mathrm{k}}$, as one may expect.

Let us first notice that we find almost all the models to be in the sensitivity regime of both LIGO and EPTA. There is indeed a significant amount of power around the LIGO frequency $f_{0}=25 \mathrm{~Hz}$ coming from the peak associated with the gravitational backreaction length scale, an effect neglected so far. Interestingly enough, we find that the constraints set by LIGO and EPTA on $G U$ for the smooth model $2 \mathrm{C}$ are within an order of magnitude range, LIGO being slightly less constraining than EPTA. The moderately kinky model, LNK, falls short under the LIGO sensitivity threshold but is well constrained by EPTA. On the contrary, the very kinky loop model HNK is strongly constrained by LIGO, and to a lesser extend by EPTA. These results should make clear the complementarity of using two very different frequencies. A closer look to figure 9 for LIGO reveals a change of behaviour in the 

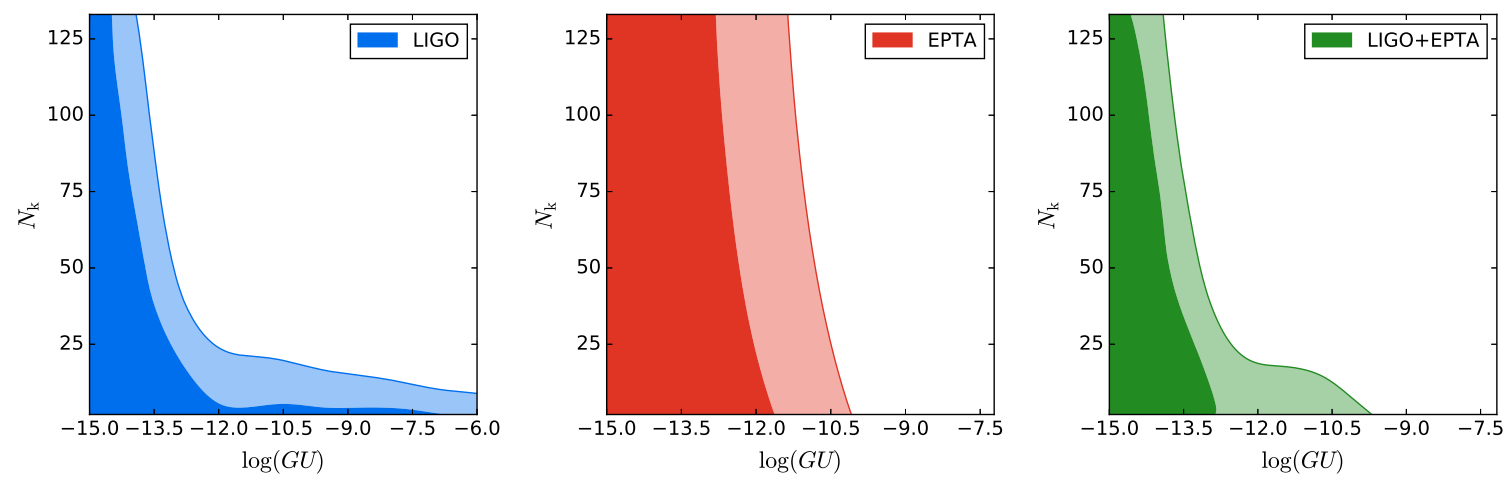

Figure 12. One- and two-sigma contours of the posterior probability distributions in the plane $\left[N_{\mathrm{k}}, \log (G U)\right]$ for the model MIX having only two cusps for very small loops $\left(\gamma<\gamma_{\mathrm{c}}\right)$ and $N_{\mathrm{k}}$ kinks for the larger loops. This scenario is severely constrained by EPTA while LIGO is sensitive to values of $N_{\mathrm{k}} \gg 20$ only.

\begin{tabular}{|c|l|l|l|}
\hline Model & LIGO & EPTA & LIGO + EPTA \\
\hline 2C & $G U \leq 1.1 \times 10^{-10}$ & $G U \leq 3.4 \times 10^{-11}$ & $G U \leq 1.0 \times 10^{-11}$ \\
\hline LNK & - & $G U \leq 6.8 \times 10^{-11}$ & $G U \leq 7.2 \times 10^{-11}$ \\
\hline HNK & $G U \leq 8.8 \times 10^{-14}$ & $G U \leq 6.4 \times 10^{-12}$ & $G U \leq 6.7 \times 10^{-14}$ \\
\hline MIX & $G U \leq 1.4 \times 10^{-8}$ & $G U \leq 1.1 \times 10^{-11}$ & $G U \leq 5.9 \times 10^{-12}$ \\
\hline
\end{tabular}

Table 1. Two-sigma upper limit for the string tension $G U$ associated with the prototypical models of string microstructure. For the two cusps model $(2 \mathrm{C}), G U$ is the only parameter whereas for the kinky models, LNK and HNK, and the scale-dependent model MIX, the constraints are obtained by marginalisation over the number of kinks $N_{\mathrm{k}}$. The EPTA limits apply to all the scenarios whereas LIGO ends up being constraining 2C and HNK. For the MIX model, the LIGO bound on GU exists only at two-sigma and disappears at three sigma (see text).

tail of the posterior distribution. This feature can be understood from figure 5. By lowering $G U$, the peak of the spectrum moves to higher frequencies and, combined with thermal history effects, around $f=10 \mathrm{~Hz}$, this can actually induce a transient increase in power.

Finally, the MIX model is slightly constrained by LIGO and much constrained by EPTA. As can be seen in figure 7, the low-frequency part of the spectrum associated with MIX is dominated by kinks only. As a result, the EPTA constraint on $G U$ for MIX ends up being in between the one associated with LNK and HNK. Around the LIGO frequencies, cusps are present but their overall contribution to the spectrum remains smaller than the one coming from kinks and collisions, at least for most of the possible values of $N_{\mathrm{k}}$. In figure 9 , one can see that the tail of the one-dimensional posterior distribution for $G U$ is not vanishing. From the two-dimensional posterior of figure 12, it comes from the low values of $N_{\mathrm{k}}$ which fall under the sensitivity threshold of LIGO, as it is the case for LNK. As a result, we find that there is only a two-sigma LIGO bound on $G U \leq 1.4 \times 10^{-8}$, and no constraint at all at three-sigma.

From the one-dimensional marginalised probability distributions, we have extracted the two-sigma upper limits on the string tension $G U$ for each scenario. They have been reported in Table. 1. 


\section{Conclusion}

We have provided a new estimation of the stochastic GW spectrum generated by a network of cosmic strings in scaling. The loop distribution underlying our calculations comes from Nambu-Goto simulations and our Boltzmann approach allows us to consider any relaxation effects that are associated with the transition from the radiation era to the matter era. Most importantly, we have also included both the effect of GW emission at $\gamma \leq \gamma_{d}$ and GW backreaction at $\gamma \leq \gamma_{\mathrm{c}}$ on the loop distribution. The fact that these scales do not match yields two characteristic features in the spectrum which may not have been appreciated enough before.

Because some theoretical uncertainties remain on the loop microstructure which can survive the cosmological history, we have considered various motivated possibilities in which cosmic string loops may, or may not, have a high number of kinks instead of the usual cusps. In particular, we have shown that kinky loops produce a stochastic gravitational wave spectrum dominated by the collision of left- and right-moving kinks, an effect neglected so far. Let us also mention that, as discussed in Refs. [77], one may also expect some electromagnetic signatures in this regime.

In order to estimate how much the string microstructure affects the stochastic GW spectrum, we have devised four prototypical models. The first $(2 \mathrm{C})$ corresponds to loops developing two cusps per oscillation, it is a smooth string model corresponding to the standard lore. We have also considered loops having a low number of kinks $N_{\mathrm{k}} \leq 20$ (LNK) and loops having a higher number of kinks $20<N_{\mathrm{k}} \leq 133$ (HNK). In the latter case, the overall stochastic GW spectrum is expected to be dominated by collision events. Another model, MIX, is a mixture of the $2 \mathrm{C}$, LNK and HNK and describes small smooth loops with two cusps if their size $\gamma \leq \gamma_{\mathrm{c}}$ whereas all larger loops $\gamma>\gamma_{\mathrm{c}}$ are assumed to have only kinks, with $0 \leq N_{\mathrm{k}} \leq 133$.

The stochastic GW spectrum for these four prototypical scenarios has been compared to the current LIGO and EPTA bounds. For this purpose, we have used a nested sampling exploration of the two-dimensional parameter space associated with the string tension $G U$ and the number of kinks $N_{\mathrm{k}}$. By marginalizing over the number of kinks, we have extracted the two-sigma upper limits on the string tension $G U$, for each of the scenario. The results have been summarized in Table. 1. All the scenarios are constrained by the joined LIGO and EPTA limits on the stochastic GW background, the smooth and moderately kinky models (MIX included) having $G U \leq \mathcal{O}(1) \times 10^{-11}$ at two-sigma level while the very kinky one satisfies $G U \leq \mathcal{O}(1) \times 10^{-14}$. Let us stress again the complementarity of using both LIGO and EPTA which are probing different frequency domains. The two cusps (2C), the low number of kinks (LNK) and the mixture (MIX) models are more constrained by the EPTA bounds in the low frequency part of the spectrum whereas the high number of kinks scenario (HNK) is already severely bounded by LIGO alone. The LIGO frequency domain is indeed matching the peak of the string-generated GW background when GW backreaction on the loop distribution is considered. According to Ref. [70], the design sensitivity of LIGO in this frequency could yield a two orders of magnitude improvement on $\Omega_{\alpha}$, and thus on $G U$. Let us also stress that if stochastic GW end up being detected by LIGO, the frequency range could be exactly right to the peak, precisely in the region for which the spectral index of the spectrum is given by the loop microstructure. One would therefore know if cosmic string loops are made of cusps, kinks or collisions. 


\section{Acknowledgments}

It is a pleasure to thank Mairi Sakellariadou and Daniele Steer for providing original motivations for this work as well as the participants and organisers of the 2015 eLISA Cosmology Working Group workshop for enlightening discussions. This work was supported in part by JSPS Grant-in-Aid for Scientific Research No. 15K17632 (T. S.), and MEXT KAKENHI Grant Numbers 17H06357 (T. S.) and 17H06358 (T. S.).

\section{Note added}

On the day before this paper was submitted, two other papers appeared online, 1709.02434 and 1709.02693, providing new constraints on the cosmic string tension $G U$. They consider only a smooth loop model, and this would fit within our " $2 \mathrm{C}$ " scenario. Up to an order one factor, their constraint indeed matches ours for the Pulsar Timing Array data and the $2 \mathrm{C}$ model. This is expected as the low frequency part of our spectrum is the same as the one of Ref. [37]. However, they do not find any constraint from LIGO, probably due to their simplifying assumption of matching the scale of gravitational wave emission and gravitational wave backreaction.

\section{References}

[1] D. Kirzhnits and A. Linde, Macroscopic consequences of the Weinberg model, Phys. Lett. B 42 (Dec., 1972) 471-474.

[2] T. W. B. Kibble, Topology of cosmic domains and strings., J. Phys. A 9 (1976) 1387-1398.

[3] E. Witten, Cosmic Superstrings, Phys. Lett. B153 (1985) 243.

[4] G. R. Dvali and S. H. H. Tye, Brane inflation, Phys. Lett. B450 (1999) 72-82, [hep-ph/9812483].

[5] M. B. Hindmarsh and T. W. B. Kibble, Cosmic strings, Rept. Prog. Phys. 58 (1995) 477-562, [hep-ph/9411342].

[6] R. Durrer, M. Kunz and A. Melchiorri, Cosmic structure formation with topological defects, Phys. Rep. 364 (June, 2002) 1-81, [astro-ph/0110348].

[7] J. Polchinski, Introduction to cosmic F- and D-strings, hep-th/0412244.

[8] A.-C. Davis, P. Brax and C. van de Bruck, Brane Inflation and Defect Formation, Phil. Trans. Roy. Soc. Lond. A366 (2008) 2833-2842, [0803.0424].

[9] E. J. Copeland and T. W. B. Kibble, Cosmic Strings and Superstrings, Proc. Roy. Soc. Lond. A466 (2010) 623-657, [0911.1345].

[10] M. Sakellariadou, Cosmic Strings and Cosmic Superstrings, Nucl. Phys. Proc. Suppl. 192-193 (2009) 68-90, [0902.0569].

[11] C. Ringeval, Cosmic strings and their induced non-Gaussianities in the cosmic microwave background, Adv. Astron. 2010 (2010) 380507, [1005.4842].

[12] A. Albrecht and N. Turok, Evolution of cosmic string networks, Phys. Rev. D40 (Aug., 1989) 973-1001.

[13] D. P. Bennett and F. R. Bouchet, Cosmic-string evolution, Phys. Rev. Lett. 63 (Dec., 1989) $2776-2779$.

[14] B. Allen and P. Shellard, Cosmic-string evolution - A numerical simulation, Phys. Rev. Lett. 64 (Jan., 1990) 119-122. 
[15] D. P. Bennett and F. R. Bouchet, High-resolution simulations of cosmic-string evolution. I. Network evolution, Phys. Rev. D41 (Apr., 1990) 2408-2433.

[16] A. Vilenkin, Gravitation radiation from cosmic strings., Phys. Lett. B 107 (1981) 47-50.

[17] C. J. Hogan and M. J. Rees, Gravitational interactions of cosmic strings, Nature 311 (1984) 109-113.

[18] F. S. Accetta and L. M. Krauss, The stochastic gravitational wave spectrum resulting from cosmic string evolution, Nucl. Phys. B319 (1989) 747-764.

[19] M. Sakellariadou, Gravitational waves emitted from infinite strings, Phys. Rev. D42 (1990) 354-360.

[20] D. P. Bennett and F. R. Bouchet, Constraints on the gravity wave background generated by cosmic strings, Phys. Rev. D43 (1991) 2733-2735.

[21] G. Vincent, N. D. Antunes and M. Hindmarsh, Numerical Simulations of String Networks in the Abelian-Higgs Model, Phys. Rev. Lett. 80 (Mar., 1998) 2277-2280, [hep-ph/9708427].

[22] J. N. Moore, E. P. S. Shellard and C. J. A. P. Martins, Evolution of Abelian-Higgs string networks, Phys. Rev. D65 (Jan., 2001) 023503, [hep-ph/0107171].

[23] M. Hindmarsh, S. Stuckey and N. Bevis, Abelian Higgs Cosmic Strings: Small Scale Structure and Loops, Phys. Rev. D79 (2009) 123504, [0812.1929].

[24] M. Hindmarsh, J. Lizarraga, J. Urrestilla, D. Daverio and M. Kunz, Scaling from gauge and scalar radiation in Abelian Higgs string networks, 1703.06696.

[25] C. Ringeval, M. Sakellariadou and F. Bouchet, Cosmological evolution of cosmic string loops, JCAP 0702 (2007) 023, [astro-ph/0511646].

[26] V. Vanchurin, K. Olum and A. Vilenkin, Cosmic string scaling in flat space, Phys. Rev. D72 (2005) 063514, [gr-qc/0501040].

[27] C. J. A. P. Martins and E. P. S. Shellard, Fractal properties and small-scale structure of cosmic string networks, Phys. Rev. D73 (2006) 043515, [astro-ph/0511792].

[28] R. R. Caldwell and B. Allen, Cosmological constraints on cosmic string gravitational radiation, Phys. Rev. D45 (1992) 3447-3468.

[29] T. Damour and A. Vilenkin, Gravitational wave bursts from cosmic strings, Phys. Rev. Lett. 85 (2000) 3761-3764, [gr-qc/0004075].

[30] M. R. DePies and C. J. Hogan, Stochastic Gravitational Wave Background from Light Cosmic Strings, Phys. Rev. D75 (2007) 125006, [astro-ph/0702335].

[31] T. Regimbau, S. Giampanis, X. Siemens and V. Mandic, The stochastic background from cosmic (super)strings: popcorn and (Gaussian) continuous regimes, Phys. Rev. D85 (2012) 066001, [1111.6638].

[32] P. Binetruy, A. Bohe, C. Caprini and J.-F. Dufaux, Cosmological Backgrounds of Gravitational Waves and eLISA/NGO: Phase Transitions, Cosmic Strings and Other Sources, JCAP 1206 (2012) 027, [1201.0983].

[33] S. Kuroyanagi, K. Miyamoto, T. Sekiguchi, K. Takahashi and J. Silk, Forecast constraints on cosmic string parameters from gravitational wave direct detection experiments, Phys. Rev. D86 (2012) 023503, [1202.3032].

[34] VIRGO, LIGO Scientific collaboration, J. Aasi et al., Constraints on cosmic strings from the LIGO-Virgo gravitational-wave detectors, Phys. Rev. Lett. 112 (2014) 131101, [1310.2384].

[35] S. Henrot-Versille et al., Improved constraint on the primordial gravitational-wave density using recent cosmological data and its impact on cosmic string models, Class. Quant. Grav. 32 (2015) 045003, [1408.5299]. 
[36] L. Sousa and P. P. Avelino, Probing Cosmic Superstrings with Gravitational Waves, Phys. Rev. D94 (2016) 063529, [1606.05585].

[37] J. J. Blanco-Pillado, K. D. Olum and B. Shlaer, The number of cosmic string loops, Phys.Rev. D89 (2014) 023512, [1309.6637].

[38] T. Vachaspati and A. Vilenkin, Gravitational Radiation from Cosmic Strings, Phys. Rev. D31 (1985) 3052.

[39] B. Allen and E. P. S. Shellard, Gravitational radiation from cosmic strings, Phys. Rev. D 45 (Mar, 1992) 1898-1912.

[40] Planck collaboration, P. A. R. Ade et al., Planck 2013 results. XXV. Searches for cosmic strings and other topological defects, Astron. Astrophys. 571 (2014) A25, [1303.5085].

[41] A. Lazanu and P. Shellard, Constraints on the Nambu-Goto cosmic string contribution to the $C M B$ power spectrum in light of new temperature and polarisation data, JCAP 1502 (2015) 024, [1410.5046].

[42] J. Lizarraga, J. Urrestilla, D. Daverio, M. Hindmarsh and M. Kunz, New CMB constraints for Abelian Higgs cosmic strings, JCAP 1610 (2016) 042, [1609.03386].

[43] T. Damour and A. Vilenkin, Gravitational wave bursts from cusps and kinks on cosmic strings, Phys. Rev. D64 (2001) 064008, [gr-qc/0104026].

[44] M. J. Stott, T. Elghozi and M. Sakellariadou, Gravitational Wave Bursts from Cosmic String Cusps and Pseudocusps, 1612.07599.

[45] J. J. Blanco-Pillado, K. D. Olum and B. Shlaer, Cosmic string loop shapes, Phys. Rev. D92 (2015) 063528, [1508.02693].

[46] J. M. Wachter and K. D. Olum, Gravitational smoothing of kinks on cosmic string loops, Phys. Rev. Lett. 118 (2017) 051301, [1609.01153].

[47] J. M. Wachter and K. D. Olum, Gravitational backreaction on piecewise linear cosmic string loops, Phys. Rev. D95 (2017) 023519, [1609.01685].

[48] X. Siemens and K. D. Olum, Gravitational radiation and the small-scale structure of cosmic strings, Nucl. Phys. B611 (2001) 125-145, [gr-qc/0104085].

[49] X. Siemens, K. D. Olum and A. Vilenkin, On the size of the smallest scales in cosmic string networks, Phys. Rev. D66 (2002) 043501, [gr-qc/0203006].

[50] J. Polchinski and J. V. Rocha, Cosmic string structure at the gravitational radiation scale, Phys. Rev. D75 (2007) 123503, [gr-qc/0702055].

[51] M. Hindmarsh, C. Ringeval and T. Suyama, The CMB temperature trispectrum of cosmic strings, Phys. Rev. D81 (2010) 063505, [0911.1241].

[52] L. Lorenz, C. Ringeval and M. Sakellariadou, Cosmic string loop distribution on all length scales and at any redshift, JCAP 1010 (2010) 003, [1006.0931].

[53] P. Peter and C. Ringeval, A Boltzmann treatment for the vorton excess problem, JCAP 1305 (2013) 005, [1302.0953].

[54] J. V. Rocha, Scaling solution for small cosmic string loops, Phys. Rev. Lett. 100 (2008) 071601, [0709.3284].

[55] J. Polchinski and J. V. Rocha, Analytic study of small scale structure on cosmic strings, Phys. Rev. D74 (2006) 083504, [hep-ph/0606205].

[56] F. Dubath, J. Polchinski and J. V. Rocha, Cosmic String Loops, Large and Small, Phys. Rev. D77 (2008) 123528, [0711.0994].

[57] M. Hindmarsh and O. Philipsen, WIMP dark matter and the QCD equation of state, Phys. Rev. D71 (2005) 087302, [hep-ph/0501232]. 
[58] X. Siemens et al., Gravitational wave bursts from cosmic (super)strings: Quantitative analysis and constraints, Phys. Rev. D73 (2006) 105001, [gr-qc/0603115].

[59] P. Binetruy, A. Bohe, T. Hertog and D. A. Steer, Gravitational wave signatures from kink proliferation on cosmic (super-) strings, Phys. Rev. D82 (2010) 126007, [1009.2484].

[60] A. Bohe, Gravitational power from cosmic string loops with many kinks, Phys. Rev. D84 (2011) 065016, [1103.0768].

[61] A. Vilenkin and E. P. S. Shellard, Cosmic Strings and Other Topological Defects. Cambridge University Press, Cambridge, England, July, 2000.

[62] X. Siemens, V. Mandic and J. Creighton, Gravitational wave stochastic background from cosmic (super)strings, Phys. Rev. Lett. 98 (2007) 111101, [astro-ph/0610920].

[63] S. Olmez, V. Mandic and X. Siemens, Gravitational-Wave Stochastic Background from Kinks and Cusps on Cosmic Strings, Phys.Rev. D81 (2010) 104028, [1004.0890].

[64] T. Hahn, CUBA: A Library for multidimensional numerical integration, Comput. Phys. Commun. 168 (2005) 78-95, [hep-ph/0404043].

[65] T. Hahn, Concurrent Cuba, J. Phys. Conf. Ser. 608 (2015) 012066, [1408.6373].

[66] G. Blanger, F. Boudjema, A. Pukhov and A. Semenov, micrOMEGAs4.1: two dark matter candidates, Comput. Phys. Commun. 192 (2015) 322-329, [1407.6129].

[67] Y. Watanabe and E. Komatsu, Improved Calculation of the Primordial Gravitational Wave Spectrum in the Standard Model, Phys. Rev. D73 (2006) 123515, [astro-ph/0604176].

[68] S. Kuroyanagi, C. Ringeval and T. Takahashi, Early universe tomography with CMB and gravitational waves, Phys. Rev. D87 (2013) 083502, [1301.1778].

[69] S. A. Sanidas, R. A. Battye and B. W. Stappers, Constraints on cosmic string tension imposed by the limit on the stochastic gravitational wave background from the European Pulsar Timing Array, Phys. Rev. D85 (2012) 122003, [1201.2419].

[70] Virgo, Ligo Scientific collaboration, B. P. Abbott et al., Upper Limits on the Stochastic Gravitational-Wave Background from Advanced LIGOs First Observing Run, Phys. Rev. Lett. 118 (2017) 121101, [1612.02029].

[71] J. P. W. Verbiest et al., The International Pulsar Timing Array: First Data Release, Mon. Not. Roy. Astron. Soc. 458 (2016) 1267-1288, [1602.03640].

[72] F. Feroz and M. P. Hobson, Multimodal nested sampling: an efficient and robust alternative to Markov Chain Monte Carlo methods for astronomical data analyses, Mon. Not. R. Astron. Soc. 384 (Feb., 2008) 449-463, [0704.3704].

[73] R. Trotta, F. Feroz, M. P. Hobson, L. Roszkowski and R. Ruiz de Austri, The Impact of priors and observables on parameter inferences in the Constrained MSSM, JHEP 12 (2008) 024, [0809.3792].

[74] F. Feroz, M. P. Hobson and M. Bridges, MULTINEST: an efficient and robust Bayesian inference tool for cosmology and particle physics, Mon. Not. R. Astron. Soc. 398 (Oct., 2009) 1601-1614, [0809.3437].

[75] F. Feroz, M. Hobson, E. Cameron and A. Pettitt, Importance Nested Sampling and the MultiNest Algorithm, 1306.2144.

[76] C. Ringeval, Fast Bayesian inference for slow-roll inflation, Mon. Not. Roy. Astron. Soc. 439 (2014) 3253-3261, [1312.2347].

[77] D. A. Steer and T. Vachaspati, Light from Cosmic Strings, Phys. Rev. D83 (2011) 043528, [1012.1998]. 